\title{
Differential impact of RB status on E2F1 reprogramming in human cancer
}

\author{
Christopher McNair, ${ }^{1}$ Kexin Xu, ${ }^{2}$ Amy C. Mandigo, ${ }^{1}$ Matteo Benelli, ${ }^{3}$ Benjamin Leiby, ${ }^{4}$ Daniel Rodrigues, ${ }^{5}$ Johan Lindberg, ${ }^{6}$ \\ Henrik Gronberg, ${ }^{6}$ Mateus Crespo, ${ }^{5}$ Bram De Laere, ${ }^{7}$ Luc Dirix, ${ }^{7,8}$ Tapio Visakorpi, ${ }^{9}$ Fugen Li, ${ }^{10}$ Felix Y. Feng, ${ }^{11}$ Johann de Bono, ${ }^{5}$ \\ Francesca Demichelis, ${ }^{3,12}$ Mark A. Rubin, ${ }^{12,13}$ Myles Brown, ${ }^{10,14}$ and Karen E. Knudsen ${ }^{1}$
}

\begin{abstract}
'Department of Cancer Biology, Sidney Kimmel Cancer Center, Thomas Jefferson University, Philadelphia, Pennsylvania, USA. ${ }^{2}$ Department of Molecular Medicine/Institute of Biotechnology, University of Texas Health Science Center at San Antonio, San Antonio, Texas, USA. ${ }^{3}$ Centre for Integrative Biology, University of Trento, Trento, Italy. ${ }^{4}$ Department of Pharmacology and Experimental Therapeutics, Sidney Kimmel Cancer Center, Thomas Jefferson University, Philadelphia, Pennsylvania, USA. ${ }^{5}$ Division of Cancer Therapeutics and Division of Clinical Studies, The Institute of Cancer Research, London, United Kingdom. ${ }^{6}$ Department of Medical Epidemiology and Biostatistics, Karolinska Institutet, Stockholm, Sweden. ' ${ }^{7}$ entre for Oncological Research, University of Antwerp, Antwerp, Belgium. ${ }^{8}$ Department of Oncology, GZA Hospitals Sint-Augustinus, Antwerp, Belgium. ' ${ }^{B}$ BioMediTech Institute and Faculty of Medicine and Life Sciences, University of Tampere and Fimlab Laboratories, Tampere University Hospital, Tampere, Finland. ${ }^{10}$ Center for Functional Cancer Epigenetics, Dana-Farber Cancer Institute, Boston, Massachusetts, USA. "Department of Radiation Oncology, Urology, and Medicine and Helen Diller Family Comprehensive Cancer Center, UCSF, San Francisco, California, USA. ${ }^{12}$ Englander Institute for Precision Medicine, Weill Cornell Medicine and New York Presbyterian Hospital, New York, New York, USA ${ }^{13}$ Sandra and Edward Meyer Cancer Center at Weill Cornell Medicine, New York, New York, USA. ${ }^{14}$ Department of Medical Oncology, Dana-Farber Cancer Institute, Boston, Massachusetts, USA.
\end{abstract}

\begin{abstract}
The tumor suppressor protein retinoblastoma (RB) is mechanistically linked to suppression of transcription factor E2F1mediated cell cycle regulation. For multiple tumor types, loss of RB function is associated with poor clinical outcome. RB action is abrogated either by direct depletion or through inactivation of RB function; however, the basis for this selectivity is unknown. Here, analysis of tumor samples and cell-free DNA from patients with advanced prostate cancer showed that direct RB loss was the preferred pathway of disruption in human disease. While RB loss was associated with lethal disease, RB-deficient tumors had no proliferative advantage and exhibited downstream effects distinct from cell cycle control. Mechanistically, RB loss led to E2F1 cistrome expansion and different binding specificity, alterations distinct from those observed after functional RB inactivation. Additionally, identification of protumorigenic transcriptional networks specific to RB loss that were validated in clinical samples demonstrated the ability of RB loss to differentially reprogram E2F1 in human cancers. Together, these findings not only identify tumor-suppressive functions of RB that are distinct from cell cycle control, but also demonstrate that the molecular consequence of RB loss is distinct from RB inactivation. Thus, these studies provide insight into how RB loss promotes disease progression, and identify new nodes for therapeutic intervention.
\end{abstract}

\section{Introduction}

The function of the retinoblastoma tumor suppressor (RB) in preventing tumor development relies in large part on the capacity of this transcriptional corepressor to modulate E2F family transcription factor activity $(1,2)$. The E2F family consists of 8 members, generally separated into 3 classes: typical activators (E2F1-E2F3a), canonical repressors (E2F3b-E2F6), and atypical repressors (E2F7 and E2F8) (2-4). Activator E2Fs use dimerization partners to bind DNA at promoter regions of target genes, and induce transcriptional programs that result in a myriad of outcomes, including cell cycle progression and DNA repair (5-7). In conditions favoring cell cycle arrest (e.g., nutrient deprivation), activator E2Fs are held inactive through association with RB (4), whereas pro-proliferative signals (e.g., growth factor stimulation) inactivate RB function through cyclindependent kinase-mediated (CDK-mediated) phosphorylation,

Authorship note: C. McNair and K. Xu contributed equally to this work. Conflict of interest: The authors have declared that no conflict of interest exists. Submitted: February 22, 2017; Accepted: October 24, 2017 Reference information: J Clin Invest. 2018;128(1):341-358. https://doi.org/10.1172/JCI93566. thus releasing $\mathrm{RB}$ from activator $\mathrm{E} 2 \mathrm{~F} 1$ binding and derepressing E2F1 activity $(8,9)$. While RB is capable of modulating both activator and repressor E2Fs $(2-4,10)$, clinical observations to date suggest that the tumor suppressor role of RB depends on modulation of activator (rather than atypical or repressor) E2F family members, suggesting divergent functions of the E2F transcription factors in tumorigenesis (3).

Illustrating the importance of $\mathrm{RB}$ in restraining E2F1 function, pathways that abrogate $\mathrm{RB}$-mediated $\mathrm{E} 2 \mathrm{~F}$ regulation are frequently perturbed in human tumors. In a large subset of tumors retaining $\mathrm{RB}$ expression, alterations in cyclin-CDK pathways serve to inactivate tumor suppressor function. This is achieved either via upregulation of RB-inhibiting cyclin-CDK complex components (e.g., cyclin D1, CDK4, and cyclin E)(11-16) or through loss of CDK inhibitors such as $\mathrm{p} 16^{\mathrm{INK} 4 \mathrm{~A}}$ and $\mathrm{p} 27^{\mathrm{Kip} 1}$, both of which serve as tumor suppressors in their own right through this function $(11,15-$ 23). Underscoring the importance of $\mathrm{RB}$ inactivation in promoting cancer cell phenotypes, CDKs have been identified as therapeutic targets, and clinical trials for CDK inhibitors have shown promise across cancer types (NCT01291017, non-small cell lung carcinoma; NCT00141297, lymphoma; NCT01958021, MONALEESA-2, breast cancer) $(11,24-26)$, with palbociclib (CDK4/6 inhibitor) 
A

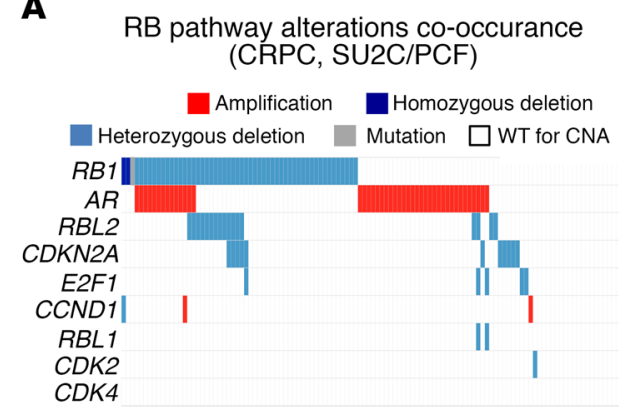

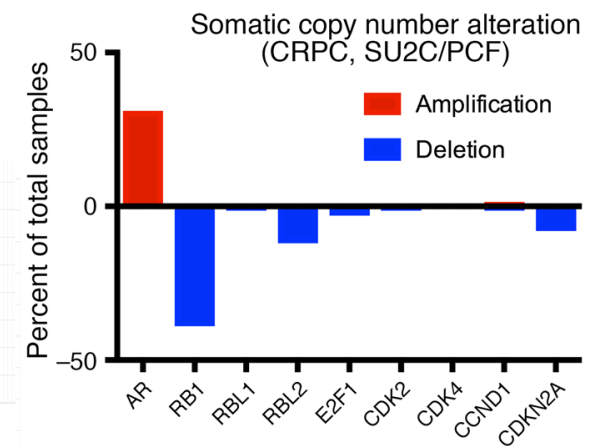
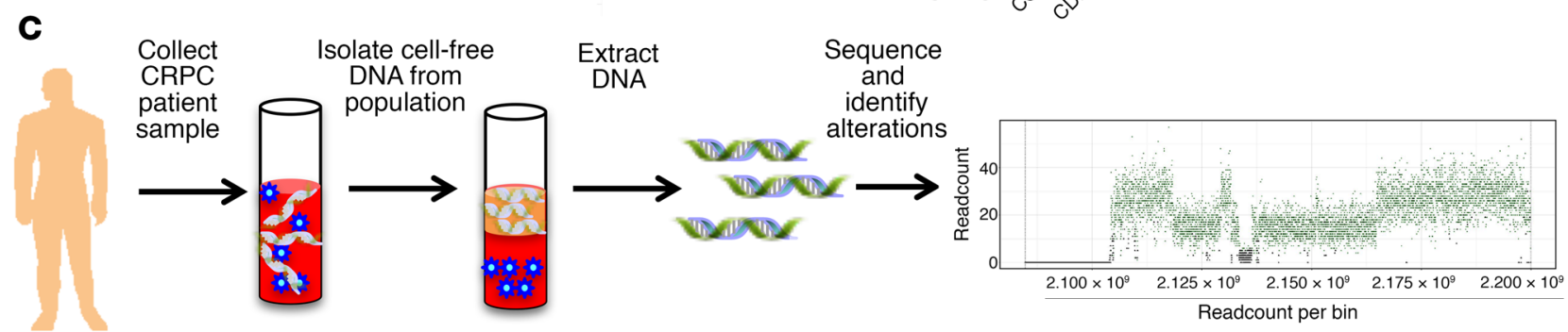

Karolinska CRPC cohort

CtDNA RB pathway alterations (Karolinksa CRPC cohort)

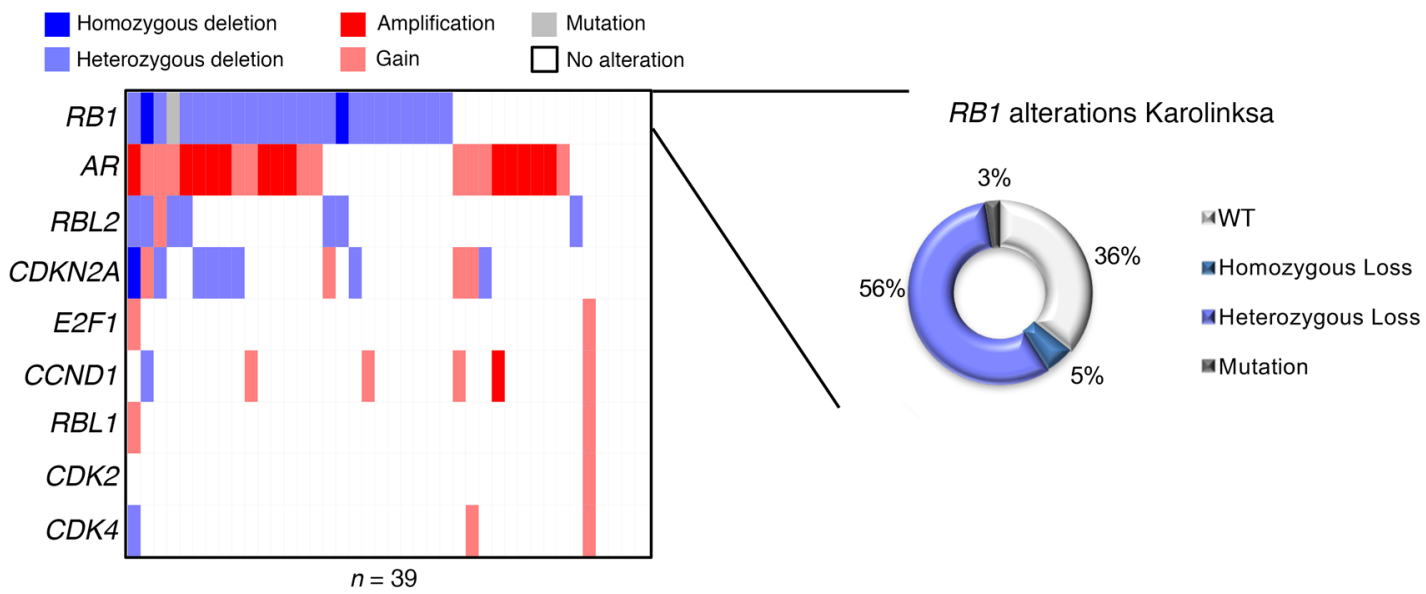

Figure 1. RB loss is frequent in CRPC and can be detected in circulating tumor DNA. (A) Mutual exclusivity plot indicating presence or absence of multiple alterations with each sample in the SU2C cohort (left) and frequency of indicated alterations in SU2C CRPC cohort ( $n=144$, right). CNA, copy number alteration. (B) Prevalence of specific RB alterations. (C) Schematic of ctDNA collection and sequencing of CRPC samples by the Karolinska Institute (top) and copy number alterations in RB pathway genes identified within the Karolinska ctDNA cohort through sequencing of a 1.3-Mb panel (bottom left) and prevalence of specific RB alterations (bottom right).

recently attaining FDA approval in hormone receptor-positive, HER2-negative, metastatic, or advanced breast cancer (27).

Distinct from tumors that preferentially retain $\mathrm{RB}$ expression but attenuate function through CDK-mediated RB inactivation, a subset of human malignancies preferentially abrogate RB through direct depletion of the protein. While tumor-specific proclivities for favoring $\mathrm{RB}$ retention and inactivation via $\mathrm{CDKs}$ versus $\mathrm{RB}$ loss have long been appreciated $(15,16,28-30)$, the underlying basis for selectivity is unknown, and the relative impact on downstream E2F1 signaling and function remains undefined. RB loss is typically achieved through genetic alterations including either genomic deletion and loss of heterozygosity or somatic mutations that generate an unstable protein $(8,15,29,30)$. Such observations are frequent in the majority of small cell lung cancers $(29,31,32)$ as well as in the genitourinary cancers $(15,29,30,33-35)$, wherein $30 \%-60 \%$ of bladder and advanced prostate cancers exhibit RB loss. In the case of prostate cancer, RB loss occurs predominantly at the time of progression from primary disease to aggressive, metastatic castration-resistant prostate cancer (CRPC) (30, 33, $34,36)$, indicating a role in promoting tumor progression rather than in tumor development.

Here, examination of RB pathway alterations in advanced prostate cancer revealed that $\mathrm{CDK} /$ cyclin/CDKi alterations are infrequent, and identified RB loss as the major mechanism of pathway disruption in human disease. Furthermore, RB status was readily traced through cell-free DNA analyses in human 
specimens, thus identifying new ways to assign RB status in the clinical setting. Strikingly, RB depletion in human disease was not associated with a higher Ki67 index, indicating a role for the $\mathrm{RB} / \mathrm{E} 2 \mathrm{~F} 1$ pathway in regulating processes distinct from cell cycle control and associated with lethal-stage disease. Subsequent mechanistic investigation used isogenic prostate cancer models, wherein $\mathrm{RB}$ could be differentially inactivated through depletion or through hormone-induced, CDK-mediated phosphorylation. Unbiased molecular interrogation uncovered a novel E2F1 cistrome and downstream engagement of transcriptional networks exclusively observed after RB loss, with binding specificity divergent from canonically described E2F1 binding patterns. Additionally, E2F1 cistrome alterations elicited by RB depletion were seen to be distinct from those after phosphorylation-induced RB functional inactivation, providing needed insight into the basis of selectivity for RB loss versus CDK-mediated inactivation observed in human disease. Analyses of human CRPC tumor samples further underscored the clinical relevance of RB loss-induced gene expression programs, which were significantly correlated with reprogrammed E2F1 binding identified herein. Taken together, the studies presented are, to our knowledge, the first to identify the consequence of $\mathrm{RB}$ loss, demonstrating molecular distinction from $\mathrm{RB}$ inactivation and illustrating the clinical relevance of $\mathrm{RB}$ loss-induced E2F rewiring.

\section{Results}

$R B / E 2 F 1$ pathway disruption is frequent in and preferentially occurs through $R B$ loss in CRPC. RB loss has been previously described to be prevalent in, enriched in, and causative for CRPC $(34,36-38)$. To expand analyses of the RB pathway to recent clinical findings, alterations in the androgen receptor (AR), RB, cyclin-CDK, and CDK inhibitor profiles were assessed using a cohort of CRPC tissue from the Stand Up to Cancer/Prostate Cancer Foundation (SU2C/PCF) prostate cancer collection (Figure 1, A and B, and ref. 39). As expected based on the known role of the AR in promoting disease progression (40), $A R$ amplification was observed in $30.5 \%(44 / 144)$ of tumors analyzed. $R B 1$ alterations were also highly prevalent, with $35.4 \%$ (51/144) exhibiting single-copy RB deletion, $1.4 \%$ (2/144) dual-copy RB deletion, and 0.7\% (1/144) RB mutation (Figure 1, A and B). Strikingly, previous tumor profiling of late-stage disease has suggested that single-allele loss of RB is sufficient to decrease RB transcript levels, supporting the significance of heterozygous RB loss observed in the current study (36). By contrast, RB-related pocket proteins p107 (RBL1) and p130 (RBL2) were rarely altered (1\% and $12 \%$, respectively; Figure $1, A$ and $B)$. There was a similar paucity of alterations in other RB pathway genes, including CDK2, CDK4, CCND1 (cyclin D1), and CDKN2A $\left(\mathrm{p} 16^{\mathrm{INK} 4 \mathrm{~A}}\right)$, demonstrating that loss of RB itself is the major mechanism of RB pathway dysfunction in CRPC. Analyses of exclusivity further demonstrated that RB pathway alterations were typically specific to loss of the $\mathrm{RB}$ locus, reinforcing the concept that $\mathrm{RB}$ pathway dysfunction is frequent and is predominantly achieved via RB loss (Figure 1, A and B).

To further assess RB pathway status in advanced disease, a second, independent cohort was examined for RB pathway alterations through isolation of cell-free tumor DNA (ctDNA) from CRPC patients, followed by sequencing of a 1.3-Mb panel (Figure
1C, top). As shown in Figure 1C, RB copy number alterations were similarly frequent, with $56.4 \%$ (22/39) exhibiting loss of a single allele, 5.1\% (2/39) loss of both alleles, and 2.5\% (1/39) RB mutation (Figure 1C, bottom). Further, with respect to alterations in $\mathrm{AR}$ as well as other RB pathway gene members, relative frequencies were similar to what was observed in the SU2C/PCF cohort (Figure 1A), suggesting that liquid biopsy via ctDNA may provide a mechanism to assess RB status in this tumor type. Together, these data indicate that genomic $\mathrm{RB}$ loss is preferentially enriched as the main mechanism of RB pathway disruption in CRPC, further underscoring the importance of $\mathrm{RB}$ loss in late-stage disease.

$R B$ loss is not associated with hyperproliferative indices. Prostate cancer is well described as a heterogeneous disease (41). As such, RB status and the extent of tumor heterogeneity were assessed in available CRPC cohorts, wherein access to tissue for immunohistochemistry (IHC) was available. Briefly, 107 CRPC tumor samples, collected by the Prostate Cancer Research Center at the University of Tampere, were examined for $R B 1$ status, AR amplification, PTEN status, and Ki67 positivity. As exemplified in Figure 2A (left) and quantified in the right panel, heterogeneity was observed in RB positivity. Tumors with the highest level of RB intensity (IHC scores 16-20 and 21-25) exhibited less than $25 \%$ as much heterogeneity as tumor cells without detectable RB, whereas a minority of tumors with reduced RB intensity (IHC scores 6-10 and 11-15, representing $21 \%$ of tumors analyzed) demonstrated the highest level of heterogeneity (Figure 2A, right). On balance, these data demonstrate that while heterogeneity in protein expression is observed, the majority of RB-positive tumors in the current cohort exhibit clear RB staining across the specimens, allowing for segregation into RBpositive versus RB-low tumor sets.

Given the known role of the RB/E2F pathway in regulating cell cycle progression (1), the impact of RB loss on proliferative indices was assessed. As shown, there was no significant correlation between Ki67 positivity and RB positivity $(\rho=0.14$, $P=0.31$; Figure $2 \mathrm{~B}$, left), suggesting that the aggressive nature of RB-deficient tumors cannot simply be attributed to a hyperproliferative phenotype. As some heterogeneity was observed for $\mathrm{RB}$ staining, weighted RB intensity score was also considered, thus taking into account variations seen within tumor samples. Further supporting a role for RB outside of proliferative control, there was no significant correlation between weighted RB score and Ki67 positivity ( $\rho=0.13, P=0.35$; Figure $2 B$, right). RB loss did not correlate with other clinically relevant alterations including $A R$ amplification or PTEN status (Supplemental Figure 1A; supplemental material available online with this article; https:// doi.org/10.1172/JCI93566DS1), concordant with data from the SU2C/PCF cohort (Figure 1A) wherein $A R$ amplification was largely observed in RB-intact tumors (30/44 tumors exhibiting $A R$ amplification displayed intact $\mathrm{RB}$ ). As neuroendocrine prostate cancer (NEPC) is characterized by loss of RB coupled with increased proliferative rates $(42-44)$, it is of note that tumors within this cohort did not exhibit prevalent neuroendocrine features, as confirmed through pathologic assessment. Additionally, no association was determined between treatment and either RB or Ki67 positivity, suggesting that treatment type did not significantly affect these correlates within this cohort (Supplemental 
A

\section{RB IHC}
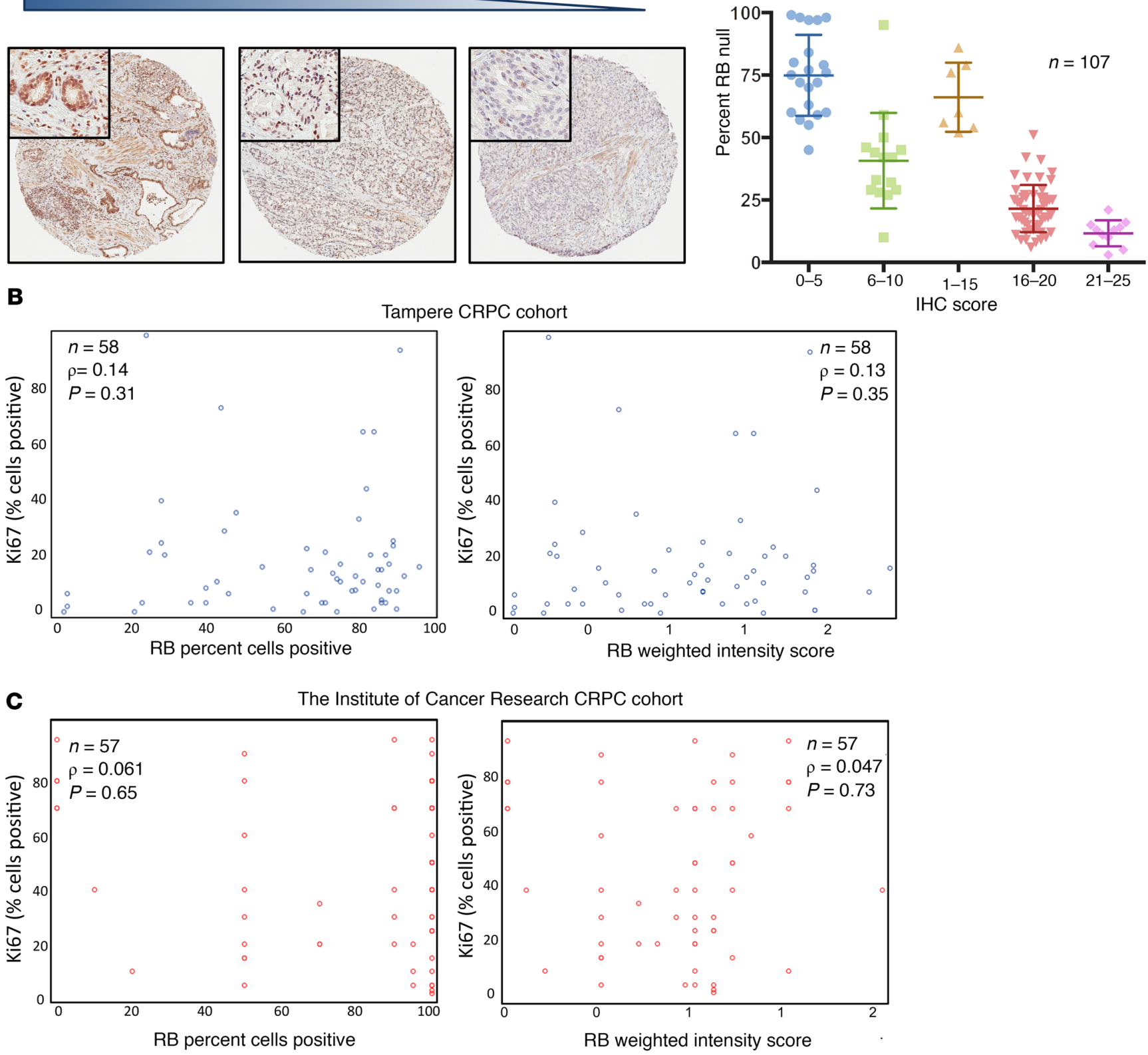

Figure 2. RB loss elicits effects outside proliferative control. (A) Left: Representative images from CRPC tissue obtained via tissue microarray (University of Tampere) through IHC staining and RB status categorized by IHC score. IHC score $=$ (frequency score $\times$ low intensity score) $+($ frequency $\times$ mid intensity score) + (frequency $\times$ high intensity score). Each core represents a $\times 6$ original magnification, and each inset a $\times 40$. Right: Grouped scatterplot illustrating quantity of RB loss within each grouping of IHC scores to illustrate RB heterogeneity. (B) Scatterplot of percent Ki67 positive versus percent of sample RB positive (left) or RB weighted intensity score (right) within the Tampere CRPC tumor cohort, with correlation shown (Spearman $\rho$ and $P$ value included). (C) Scatterplot comparing Ki67 positivity versus RB positivity (left) and weighted RB score versus percent Ki67 positive (right) within The Institute of Cancer Research CRPC tumor cohort.

Figure 1B). Finally, an additional cohort of CRPC tumors was examined for RB and Ki67 expression via IHC ( $n=57$, confirmed to include no NEPC). Confirming results in Figure 1B, this second cohort of CRPC tumors also exhibited no correlation between Ki67 positivity and RB positivity $(\rho=-0.061, P=0.65)$ or weighted RB intensity ( $\rho=0.047, P=0.73$ ), further suggesting a role for $\mathrm{RB}$ outside of canonical proliferative capacity (Figure $2 \mathrm{C}$ ). Taken together, these findings indicate that in the clinical setting, $\mathrm{RB}$ loss promotes CRPC phenotypes through mechanisms expanding beyond that of cell cycle control.

$R B$ loss results in E2F1 repositioning and expansion of the E2F1 cistrome. RB tumor suppressor function is thought to be contingent on constraining E2F transcriptional activity (1). Given the impact of RB loss on CRPC development without influencing proliferative indices, coupled with the known increases in both E2F1 transcript and protein expression after RB loss, unbiased 
genome-wide assessment of E2F1 activity was prioritized (36). For these studies, well-characterized isogenic models of RB depletion in human prostate cancer cells (shCON and shRB, Figure 3A, top left; and refs. 36, 38) were used for E2F1 chromatin immunoprecipitation followed by deep sequencing (ChIP-Seq). Statistically significant E2F1 binding was identified in each isogenic line using the model-based analysis of ChIP-Seq (MACS2) algorithm with a stringent cutoff $(Q<0.01)$. With respect to shCON cells containing intact $\mathrm{RB}$ in castrate conditions, 11,670 peaks were detected for E2F1 (Figure 3A, bottom left, red circle), consistent with previous studies of E2F1 binding in other models (Supplemental Figure $2 \mathrm{~A}$ and refs. 45,46$)$. Interestingly, upon RB knockdown in these same castrate conditions, the total number of E2F1-bound regions was substantially increased to 26,431 sites, suggesting that $\mathrm{RB}$ loss increases overall E2F1 binding capacity (Figure 3A, bottom left, blue circle). It is of note that over $90 \%$ of E2F1 binding sites observed in the presence of RB were retained after RB loss, consistent with the concept that E2F1 may exhibit gained functions upon RB loss. Further, commonly bound sites exhibited higher binding intensities upon RB knockdown, suggesting differential E2F1 binding capacity even at commonly bound sites (Figure 3A, right). Together, these data support an expansion of the E2F1 cistrome rather than a simple relocation of the existing chromatin-bound E2F1 following RB loss.

To further assess the hypothesis that RB loss rewires the E2F1 cistrome, sites of E2F1 binding gained upon RB loss were validated in an additional isogenic model of RB loss in the background of hormone therapy-sensitive LAPC4 cells (Supplemental Figure 2B). Additionally, validation was also performed in LNCaP-abl cells, which are derived from LNCaP models, but incurred loss of $\mathrm{RB}$ as a function of the transition to castration resistance (47). As shown in Supplemental Figure 2B, E2F1 binding after RB loss was validated across these models at the indicated loci, suggesting that the E2F1 rewiring displayed in LNCaP models is maintained in model systems. Further, to determine the potential effect of the mechanism by which RB is modulated on E2F1 occupancy, recently described genome-wide E2F1 binding in the LNCaP-abl model was compared with E2F1 binding in the LNCaP shRB line. Strikingly, over $70 \%$ of the shRB E2F1 cistrome identified in the current study was validated by E2F1 binding in LNCaP-abl cells, further supporting the conclusion that the expansion in E2F1 binding capacity is consistent regardless of model system or mechanism of RB modulation (Supplemental Figure 2C).

To investigate the chromosomal regions to which E2F1 was bound in each condition, cis-regulatory element annotation system (CEAS) analysis was performed. As expected, in shCON cells harboring intact RB, $49.6 \%$ of the total binding was determined to occur at promoter regions ("shCON castrate," Figure 3B). Conversely, upon RB knockdown, E2F1-bound promoter regions comprised only $26.6 \%$ of the total binding events, while distal intergenic and intronic regions consisted of $21.9 \%$ and $22.3 \%$ of the total binding, respectively ("shRB castrate," Figure $3 \mathrm{~B})$, suggesting that RB loss-induced E2F1 chromatin association may be enriched at sites other than promoters, such as enhancers. To more fully characterize the E2F1 sites gained exclusively after RB loss, CEAS analysis was performed ("shRB exclusive," 16,292 sites). Remarkably, these novel E2F1-bound regions occurred less frequently at promoter regions (15.7\%), while $31.2 \%$ of binding occurred at intronic regions and $32.6 \%$ at distal intergenic regions, further suggesting that the $\mathrm{E} 2 \mathrm{~F} 1$ sites gained upon $\mathrm{RB}$ loss largely occur outside promoter regions and may occur at enhancer regions (Figure $3 \mathrm{~B}$ ). Combined, these data provide some of the first insight into genome-wide E2F1 activity after RB loss, and demonstrate that RB depletion induces significant E2F1 cistromic reprogramming.

Given these unexpected findings, the underlying mechanisms of differential E2F1 action were investigated. De novo motif analyses were initially performed on each data set using a narrow window of $50 \mathrm{bp}$ around the center of binding to specifically highlight the characteristics of DNA sequences underlying E2F1 binding (Figure 3C, schematic). As expected, motifs closely resembling the well-described canonical E2F1 binding motif were among the top most enriched motifs in shCON E2F1-bound regions (Figure 3C, top). Interestingly, motif analysis using either the full E2F1 cistrome identified upon RB knockdown ("shRB E2F1 binding”) or the subset of E2F1-gained regions seen exclusively in the RB loss condition ("shRB exclusive E2F1 binding") showed enrichment for an abbreviated form of the canonical E2F1 motif lacking a 5' poly-T stretch (Figure 3C, middle and bottom). Importantly, recent studies have shown E2F1 to have the ability to bind this truncated, previously described minimum-affinity E2F1 motif in specific conditions (such as in the context of sustained E2F1 activity) $(46,48)$. Thus, these data suggest that in the context of intact RB, E2F1 is bound to primarily "high-affinity," canonical E2F1 motifs, while $\mathrm{RB}$ loss drives E2F1 binding to a "minimum-affinity" motif.

To further assess the means by which $\mathrm{E} 2 \mathrm{~F} 1$ is directed to new sites upon RB loss, cooperating factor motifs were investigated. For these analyses, a broader window around the center of binding (1,000 bp) was applied to the E2F1 cistromes identified herein to examine the potential for changes in cooperative factor interactions, contingent on RB status. As expected, enrichment for canonical E2F motifs was depleted upon RB knockdown (Figure 3D, left, and Supplemental Figure 3), further supporting the idea that RB loss induces E2F1 binding distinct from canonical regions. Moreover, motifs belonging to the E2F family of transcription factors represented the majority of those enriched in binding sites exclusive to the control condition (Supplemental Figure 3). Additionally, as the E2F1 cistrome exhibited a marked expansion upon $\mathrm{RB}$ loss, motifs specifically enriched in these regions were identified. To identify potential novel functional interactions, motifs with enrichment detected in control conditions were removed, leaving those exclusively associated with gained binding upon RB loss (Figure 3D, right). Strikingly, motifs enriched exclusively adjacent to E2F1 binding after RB loss consisted of several transcription factor binding motifs, including NF1, the TLX nuclear receptor, and AR. These findings not only provide the first evidence for the role that $\mathrm{RB}$ depletion may have in driving E2F1 to minimum-affinity sites, but also suggest that novel cooperating transcription factors likely assist E2F1 to mediate phenotypes associated with RB loss.

E2F1 function is distinct after RB depletion versus RB inactivation. Dihydrotestosterone (DHT) is a potent ligand for AR in prostate cancer, and has been previously shown to induce RB hyperphosphorylation and subsequent inactivation through AR-dependent 
A

E2F1 binding castrate condition

LN
shCON

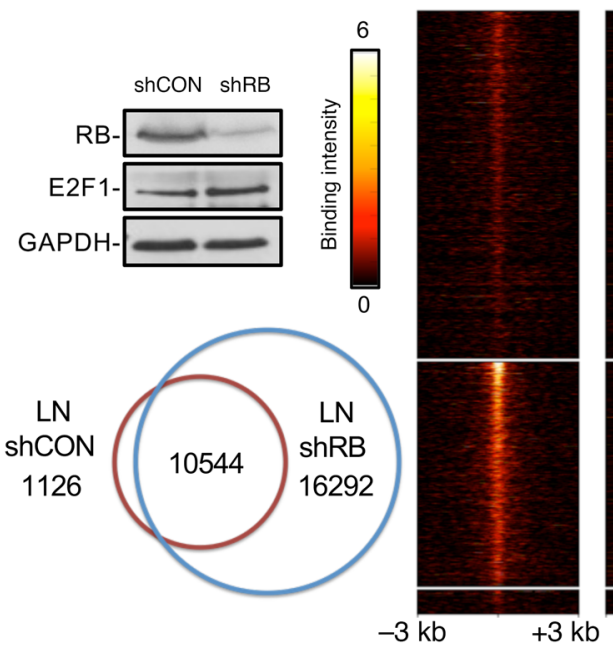

B

LN

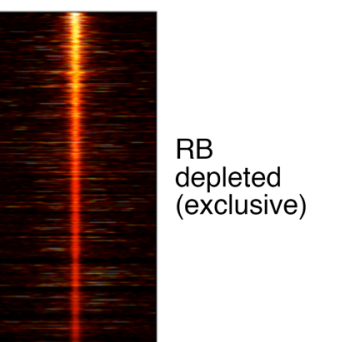

C

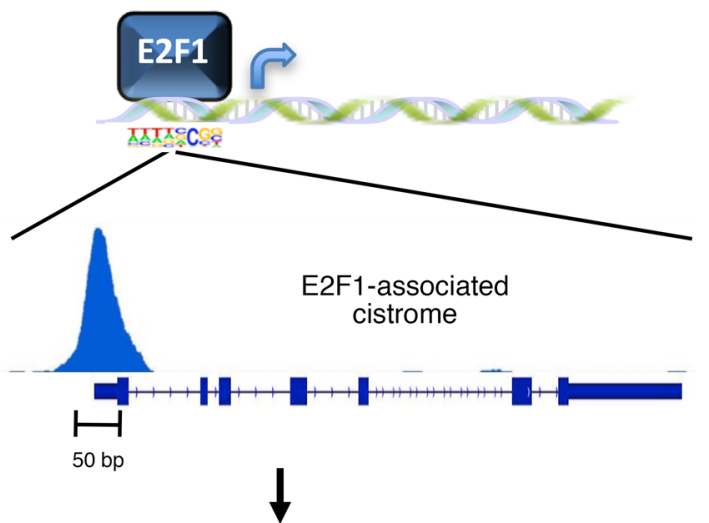

shRB E2F1 binding

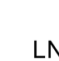
shCON

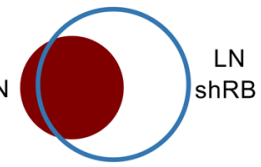

shRB E2F1 binding

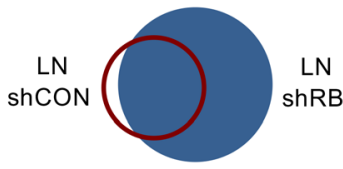

shRB exclusive E2F1 binding

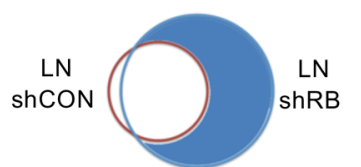

Cis-regulatory element analysis

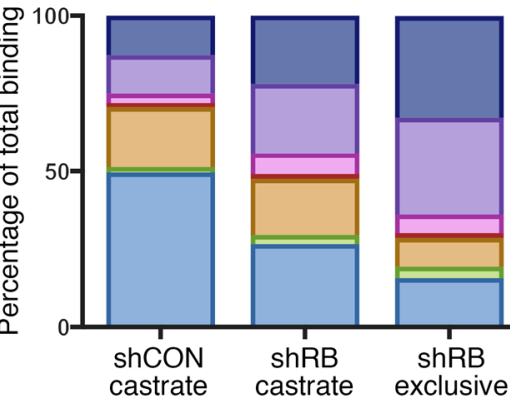

E2F1 binding data set $\square$ Distal intergenic

$\square$ Intron

$\square$ Coding exon

$\square$ 3' UTR

$\square$ 5'UTR

$\square$ Downstream

Promoter
De novo motif analysis

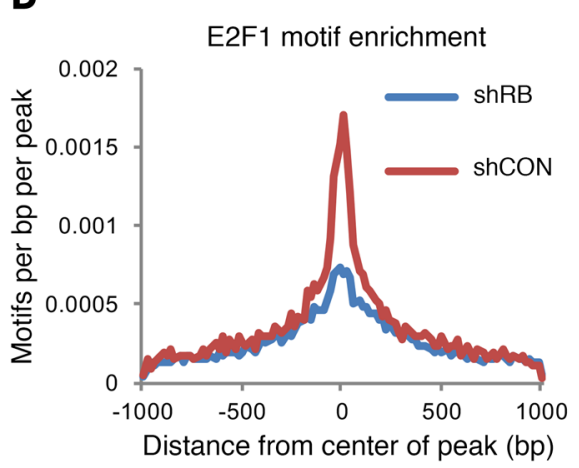

$P$ value

$1.00 \times 10^{-286}$

$1.00 \times 10^{-86}$

$P$ value

$1.00 \times 10^{-104} \mathrm{~A} \mathrm{~A} \mathrm{~A} \mathrm{C} \mathrm{C} \mathrm{A} \mathrm{AC}$ $1.00 \times 10^{-99}$

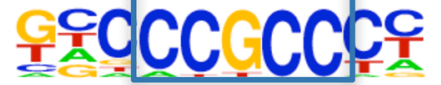

$P$ value

$1.00 \times 10^{-34}$

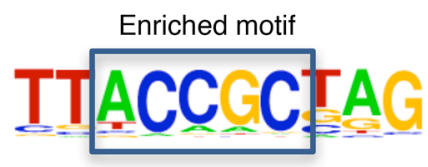

Motifs gained upon RB loss

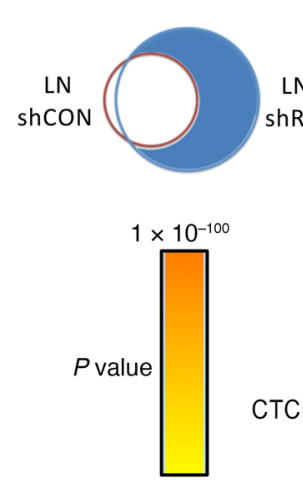

$1 \times 10^{-20}$
Enriched motif $P$ value

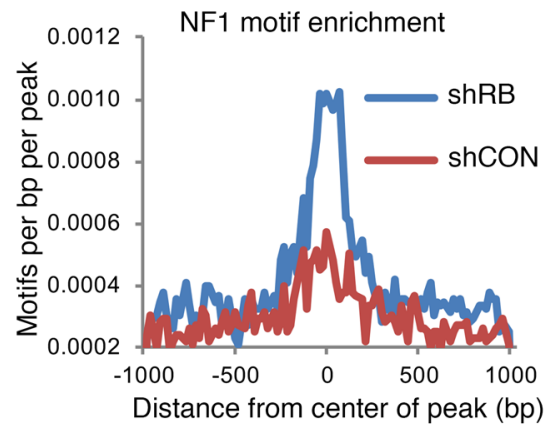


Figure 3. RB loss expands E2F1 binding capacity and repertoire of associated cis-binding elements. (A) Overlap of binding in shRB or shCON cells in castrate conditions. (B) Cis-regulatory element analysis of shCON or shRB binding data sets determined using CEAS package. Percent of total binding is shown in indicated data sets. (C) De novo motif enrichment using a 50-bp window around the center of binding. Enrichments specific to the data set are shaded in the Venn diagrams with $P$ values shown for each motif. (D) Left: JASPAR (http://jaspar.genereg.net/) E2F1 motif was used to determine enrichment in shCON E2F1 or shRB E2F1 binding data set using a 1,000-bp window around the center of binding. Right: Top motifs enriched only in shRB exclusive data set using known motifs and a 1,000-bp window around the center of binding and representative histogram of motif enrichment.

signaling $(49,50)$, thus serving as a mitogen in prostate cancer. To determine whether inactivation of RB through DHT stimulation is distinct from the changes seen upon RB depletion, E2F1 binding was assessed through additional ChIP-Seq binding studies after 3-hour DHT stimulation, which resulted in subsequent RB phosphorylation and inactivation in shCON cells (Supplemental Figure 4A). Similarly to RB knockdown, DHT stimulation resulted in an expansion of the E2F1 cistrome, with 21,510 sites gained after DHT stimulation in comparison with control (Figure 4A, orange circle and red circle, respectively). To further address the impact of hormone action on E2F1 activity, estrogen-dependent breast cancer cells were stimulated with estradiol, which has been previously shown to induce RB phosphorylation through cyclin D1/ CDK4 activity (51-54), and the impact on E2F1 was assessed on a tested subset of sites exhibiting increased E2F1 occupancy after DHT stimulation. As shown, E2F1 binding after estradiol treatment in MCF7 cells shows similar increases in occupancy, demonstrating that hormone-induced E2F1 rewiring is not exclusive to prostate cancer (Supplemental Figure 4B). Additionally, while 21,026 binding sites were commonly occupied between the RBloss (shRB castrate) and RB-inactivated (shCON 3-hour DHT) conditions, a significant fraction of E2F1 binding sites were differentially occupied in both shRB castrate conditions (5,374 sites) and RB-inactivated conditions (10,592 sites) (Figure 4A). These findings additionally underscore the concept that the molecular repercussions of RB loss are distinct from those of mitogenic stimuli-induced RB inactivation. Further, as RB loss alone in current models has been shown to significantly rewire E2F1 binding (Figure 3), the potential for androgen stimulation to further alter the E2F1 cistrome was tested. Interestingly, over $86 \%$ of sites identified in shRB models after DHT stimulation were common with those identified after RB loss alone, indicating that stimulation with androgens does not significantly alter E2F1 binding after RB loss, consistent with RB loss itself being a major driver of CRPC (Supplemental Figure 4C).

Moreover, as E2F1 binding transitioned from canonical promoter regions upon $\mathrm{RB}$ depletion (Figure $3 \mathrm{~B}$ ), changes in regulatory elements near E2F1 binding upon RB inactivation were assessed. As shown in Figure 4B, 46.4\% of E2F1 binding occurred at promoter regions upon DHT-induced RB inactivation, similar to the $49.6 \%$ bound to promoters with intact $\mathrm{RB}$ (shCON castrate), whereas only $26.6 \%$ of binding was seen at promoters upon RB depletion (shRB castrate). Consistently, only $9.5 \%$ and $9.0 \%$ of the total binding was seen at intronic and distal intergenic regions upon RB inactivation, respectively, as compared with $22.3 \%$ and
21.9\% after RB depletion. Together, these data suggest that while DHT inactivation of RB induces an increase in overall $\mathrm{E} 2 \mathrm{~F} 1$ binding, this cistrome is distinct from that seen after RB loss with respect to both overall binding site overlap and specific changes in enrichment of $c i s$-regulatory elements.

Because of the changes seen in regulatory elements associated with E2F1 occupancy between the 2 conditions, binding characteristics after RB depletion versus inactivation were assessed through de novo motif enrichment to highlight changes in DNA sequences underlying differential binding. While the CTCF binding motif was enriched in both data sets, other key distinctions were observed. Whereas the E2F1 cistrome after RB inactivation was enriched for E2F1 and FOXM motifs (Figure 4C, top right), E2F1 binding after RB depletion was most associated with nuclear receptor motifs (Figure 4C, bottom right). Together, these findings further indicate that RB inactivation through DHT stimulation and RB loss elicit divergent E2F1 binding profiles, with regard to both promoter preference and binding specificity.

Chromatin accessibility is equivalent regardless of $R B$ status. $\mathrm{RB}$ and E2F1 have both been described to interact with chromatin remodelers in various cellular contexts to alter the chromatin landscape $(48,55-58)$. Coupled with the finding that E2F1 binding is significantly expanded after RB loss, and that this expansion is distinct from E2F1 binding after DHT-induced RB inactivation, the potential for differential chromatin accessibility was assessed using assay for transposase-accessible chromatin with highthroughput sequencing (ATAC-Seq). ATAC-Seq uses hyperactive $\mathrm{Tn} 5$ transposase activity to preferentially insert adapters (used for high-throughput sequencing) in regions of open chromatin. Thus, in order to assess chromatin availability in conditions displaying the greatest changes in E2F1 binding, ATAC-Seq was performed in shRB and shCON cells in castrate conditions, as well as in shCON cells in DHT-stimulated conditions. As seen in Figure 5A, regions of open chromatin were detected across conditions in both promoter and distal regions of the indicated E2F1 targets, consistent with the capacity for this assay to identify regions of open DNA.

To detect regions of accessible DNA on a genome-wide scale, ATAC-Seq peaks were called in each condition, applying the irreproducible discovery rate (IDR) framework in order to ensure high-confidence peaks. The majority of peaks identified were present in all tested conditions tested (7,308 sites; Figure 5B, left), suggesting that while there are minor changes in open chromatin peaks observed, the chromatin landscape remains highly similar regardless of RB status or androgen stimulation. Further supporting this conclusion, normalized ATAC-Seq signal remained present across the merger of all peaks identified in all conditions, with the highest binding intensity seen in DHT-induced RB-inactivated conditions, likely due to changes outside RB/E2F1 activity, and the weakest in castrate control conditions (Figure 5B, right). Finally, principal component analysis was performed to assess variability between the conditions on a genome-wide scale. As shown in Figure 5C, each condition displays similar segregation along principal component 1 ( $x$ axis), which represents over $90 \%$ of the variance seen across the samples (Figure 5C and Supplemental Figure 5), further supporting the similarity between conditions with respect to open chromatin. Together, these data demonstrate that the overall state of chromatin accessibility remains similar after $\mathrm{RB}$ 


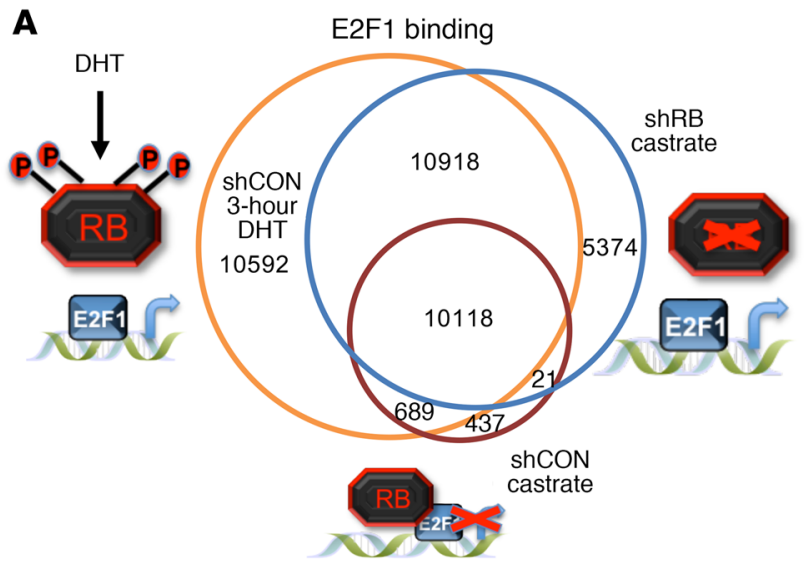

A

C

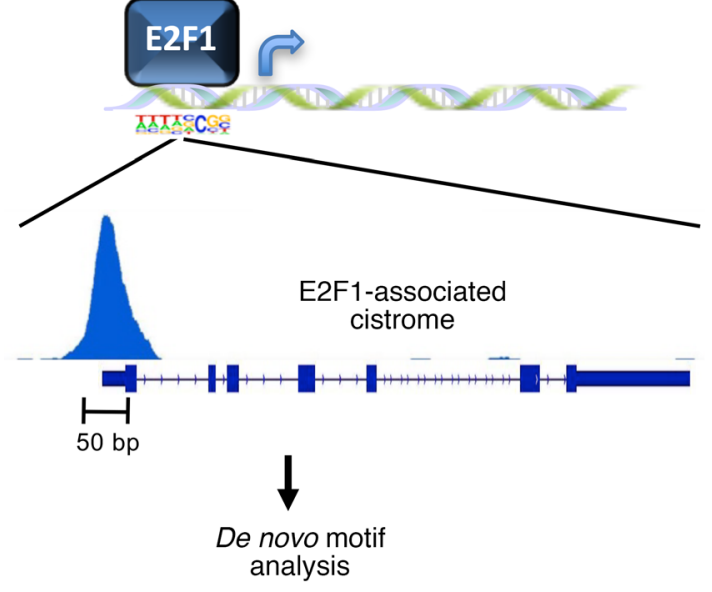

B

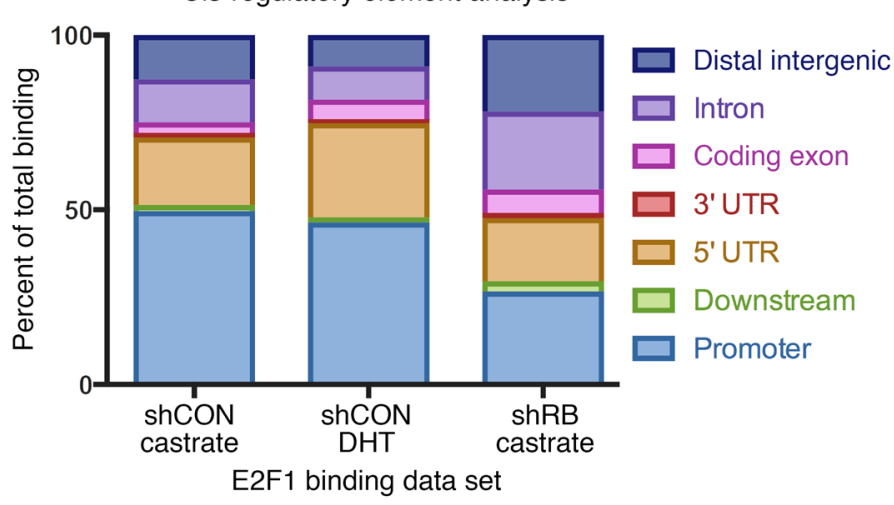

De novo motif enrichment (shCON exclusive DHT)

\begin{tabular}{|c|c|c|}
\hline$P$ value & $\begin{array}{l}\text { Nearest } \\
\text { match }\end{array}$ & Motif logo \\
\hline $1.00 \times 10^{-984}$ & CTCF & \\
\hline $1.00 \times 10^{-246}$ & FOXM1 & \\
\hline $1.00 \times 10^{-111}$ & NF1-Half & \\
\hline $1.00 \times 10^{-79}$ & $\mathrm{E} 2 \mathrm{~F} 1$ & \\
\hline
\end{tabular}

De novo motif enrichment (shRB exclusive DHT)

$P$ value Nearest Motif logo

$1.00 \times 10^{-205}$ cTCF CACCA AGTGG

$1.00 \times 10^{-47} \quad$ Tbx5 AGTGCCACCT

$1.00 \times 10^{-28} \quad$ AR-half TGCTAGCACA

$1.00 \times 10^{-27}$ Cux1 CAGGTTGATC

Figure 4. DHT-mediated RB inactivation results in distinct E2F1 reprogramming. (A) Overlap of binding in LN shCON cells in androgen-stimulated conditions (3-hour DHT) with shCON or shRB cells in castrate conditions. (B) Chromosomal enrichment analysis of castration-treated or DHT-stimulated cells, represented as percent of total binding in each indicated data set. (C) De novo motif enrichment in indicated data sets using a 50-bp window around the center of binding using binding exclusive to either the shCON DHT-stimulated condition (top) or the shRB castrate condition (bottom).

depletion or inactivation, and support the idea that E2F1 rewiring is likely not due to widespread global alterations in open chromatin and indiscriminate or opportunistic E2F1 binding.

Delineation of the RB loss-induced transcriptome. The observations that RB loss in CRPC is frequent and not correlated with a hyperproliferative phenotype (Figures 1 and 2), coupled with the finding that RB loss induces expansion of the E2F1 cistrome (Figure 3) distinct from that seen with inactivation of RB and via mechanisms outside differential chromatin accessibility (Figures 4 and 5), suggest that RB loss alters E2F1 activity in prostate cancer, and likely results in differential regulation of transcriptional networks that promote disease aggressiveness. Strikingly, there have been few reports that assess genome-wide E2F1 activity and resultant transcriptional outcomes in any tumor type, and to our knowledge no genome-wide assessment of E2F1 activity after
RB loss. As such, RNA from shCON and shRB isogenic pairs was isolated and RNA sequencing (RNA-Seq) performed. Significant transcriptional alterations were seen in castrate conditions, with 1,423 genes upregulated and 1,496 downregulated genes observed after RB loss (adjusted $P$ value $<0.05$; Figure $6 \mathrm{~A}$ ). Consistent with the role of $\mathrm{E} 2 \mathrm{~F} 1$ as a transcriptional activator, $\mathrm{RB}$ loss was seen to induce the transcription of a number of different genes, including previously described E2F1 targets such as PCNA and PLK1 (Supplemental Figure 6A). The abundance of transcripts downregulated upon RB knockdown was less anticipated, and suggests that E2F1 may directly or indirectly serve as a transcriptional repressor as well (Figure 5A, left, and Supplemental Figure 6A). To test $\mathrm{RB}$ loss-induced transcriptional changes across model systems, LAPC4 isogenic models (shCON/shRB) and LNCaP-abl models were also assessed for transcriptional changes (both up- and down- 
A

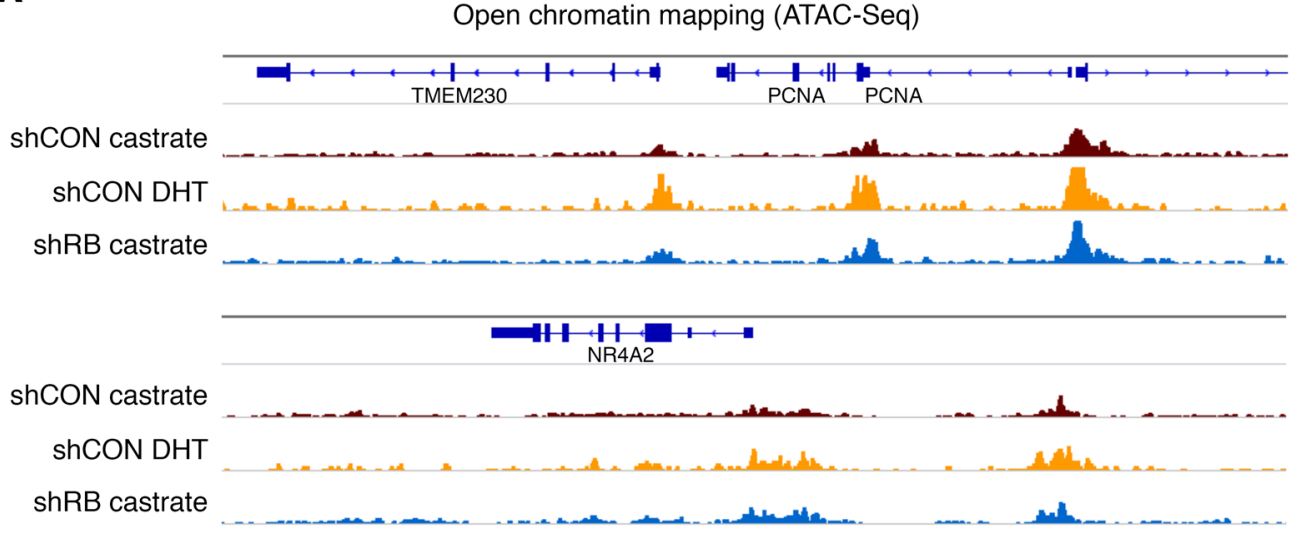

B

ATAC-Seq

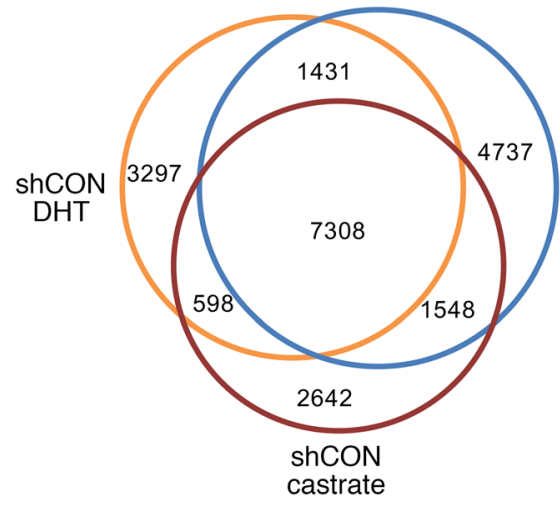

C

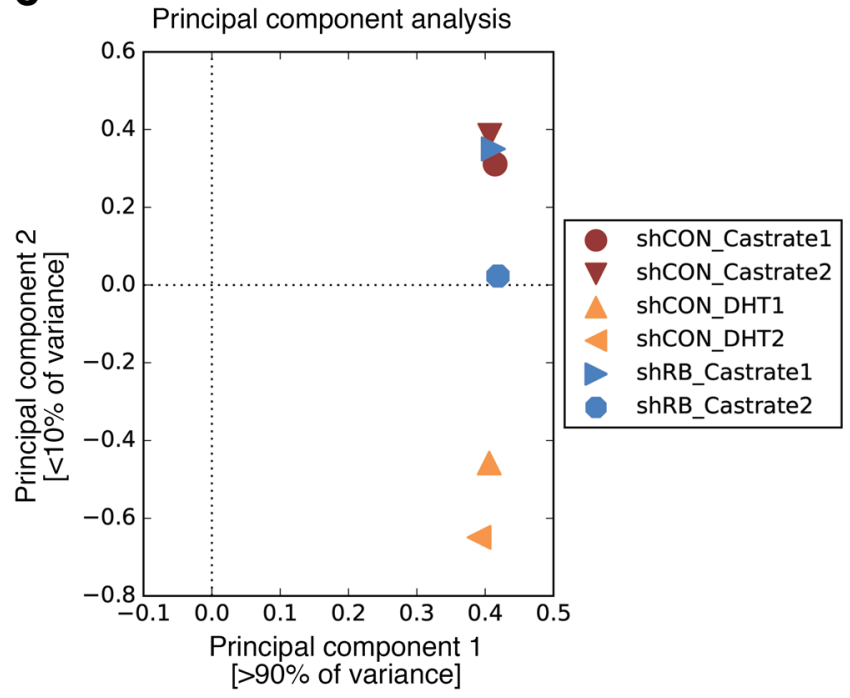

Signal across all sites

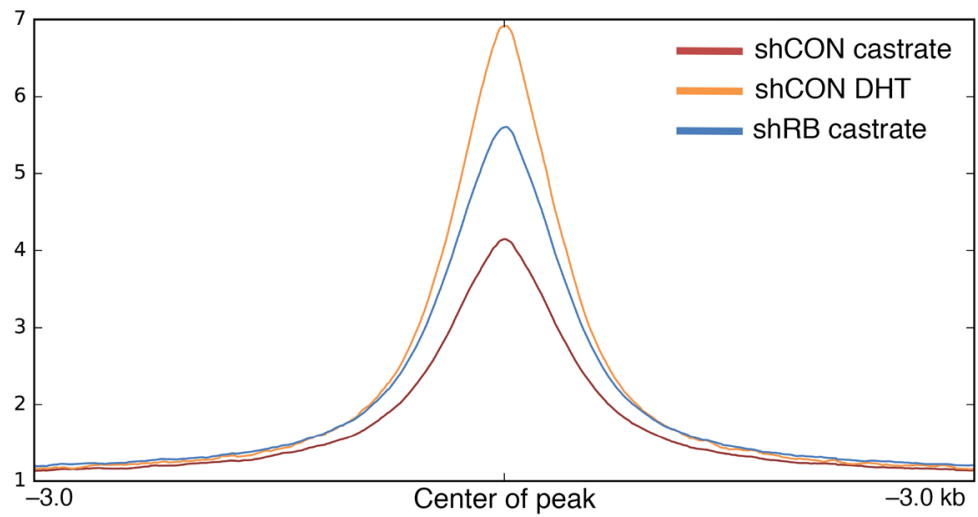

Figure 5. Chromatin landscape is consistent regardless of RB status. (A) ATAC-Seq signal at indicated loci for shCON and shRB in castrate conditions and shCON after DHT treatment using Integrative Cenomics Viewer (http://software.broadinstitute.org/software/igv/home). (B) Venn diagram representing overlap in ATAC-Seq peaks (left) and ATAC-Seq signal across all peaks in all conditions (right). Peak calling was performed using the ATAC-Seq ENCODE pipeline, and highly concordant peaks across replicates were identified using IDR and subsequently used for Venn diagram and signal profile generation. (C) Principal component analysis for ATAC-Seq replicates.

regulated) at the indicated targets. Targets assessed were seen to exhibit highly concordant changes in gene expression after RB loss, as compared with LNCaP isogenic lines, thus indicating a high degree of reproducibility across multiple model systems and mechanisms of RB loss (Supplemental Figure 6A). Additionally, to assess the potential for CDK4/6 inhibitors to enhance transcriptional alterations identified in $\mathrm{RB}$ loss versus $\mathrm{RB}$-intact conditions, shCON cells were treated with a CDK4/6 inhibitor (palbociclib), and gene expression in a subset of RB loss-induced targets was examined. As expected, no significant effect was seen on gene expression after CDK4/6 inhibitor treatment compared with castrate conditions, suggesting that castrate conditions fully activate $\mathrm{RB}$ in the context examined (Supplemental Figure 6B). Finally, as DHT stimulation was seen to have little added effect on E2F1 binding after RB loss alone (Supplemental Figure 4B), the potential for DHT to further deregulate the RB loss-induced transcriptome was tested. As shown in Figure 6B, no significant alterations were identified in shRB cells in castrate versus DHT-stimulated conditions, consistent with the supposition that RB loss itself represents the main mechanism of transcriptional deregulation. 
A

RNA-Seq

shRB castrate/shCON castrate

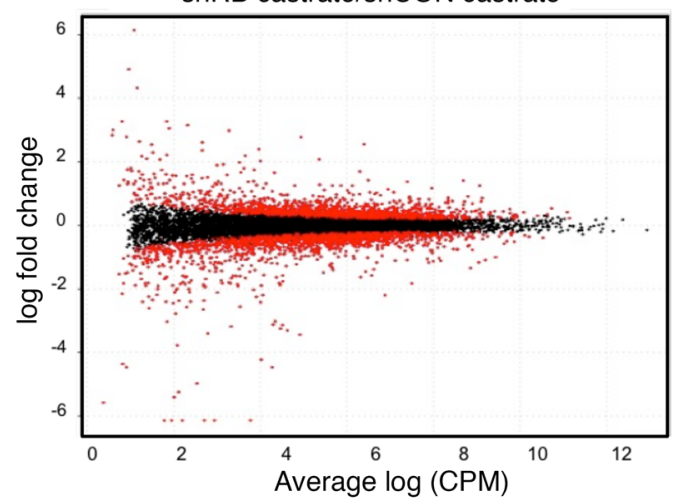

C

Pathways de-enriched after RB knockdown in castrate conditions

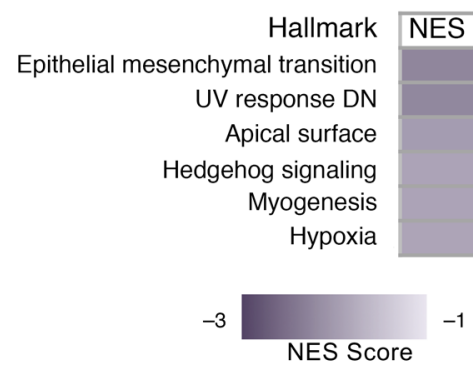

Hallmark NES

Apical surface

Myogenesis

Hypoxia

NES Score
B

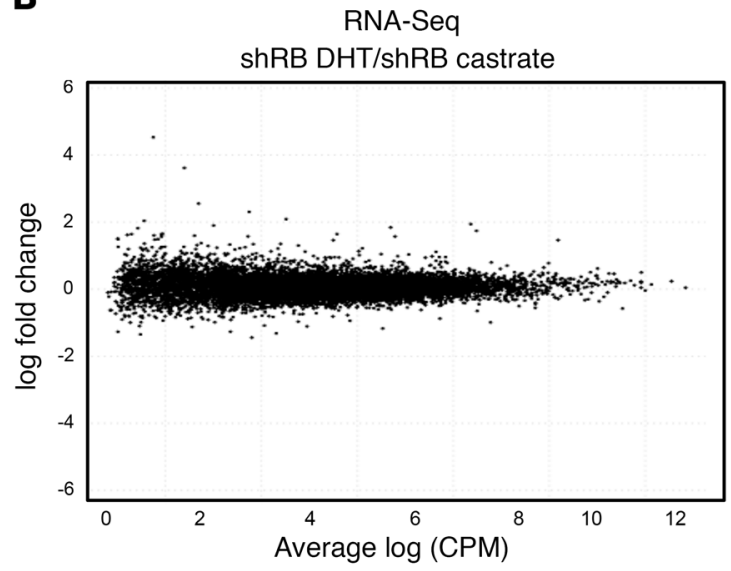

Pathways enriched after

RB knockdown in castrate conditions

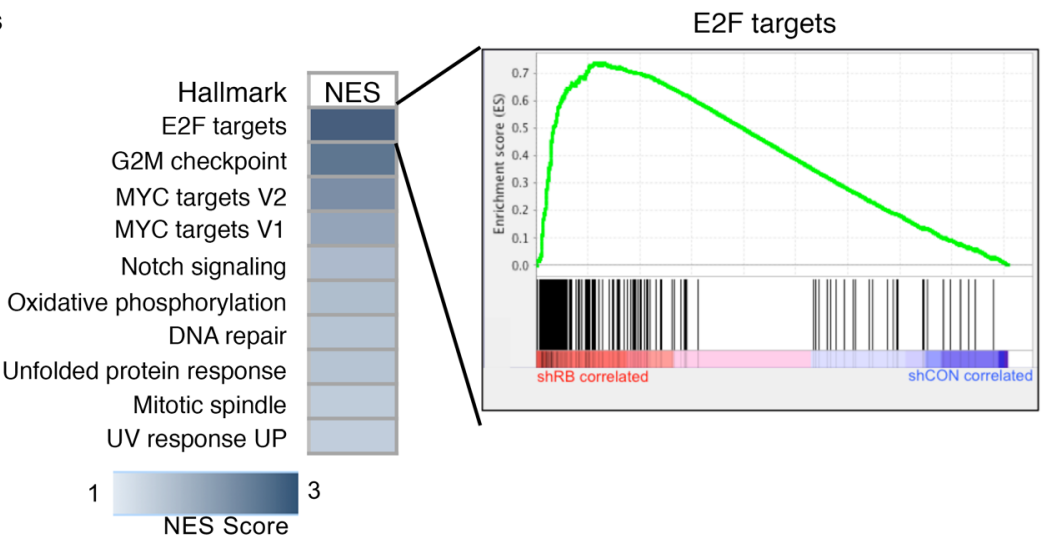

D

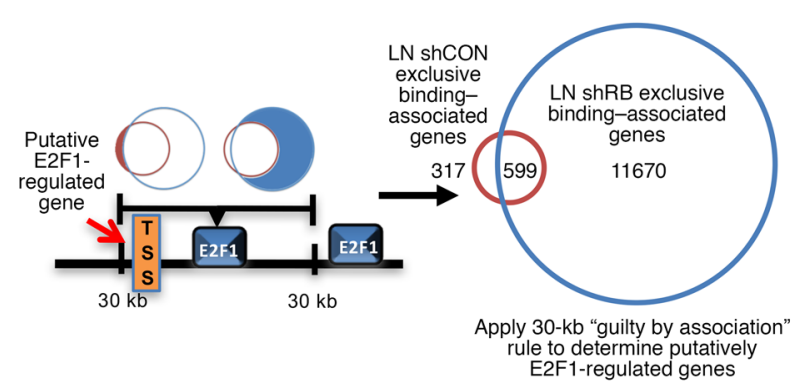

E

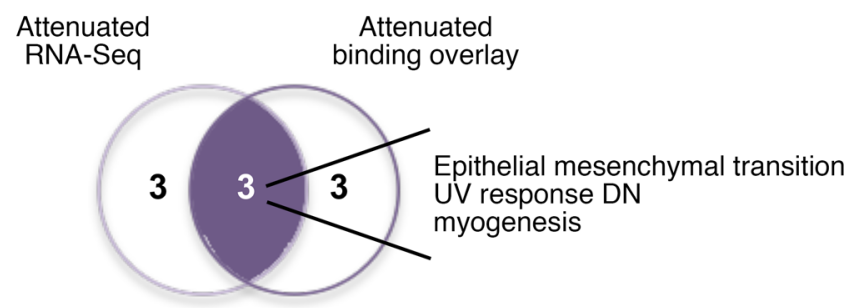

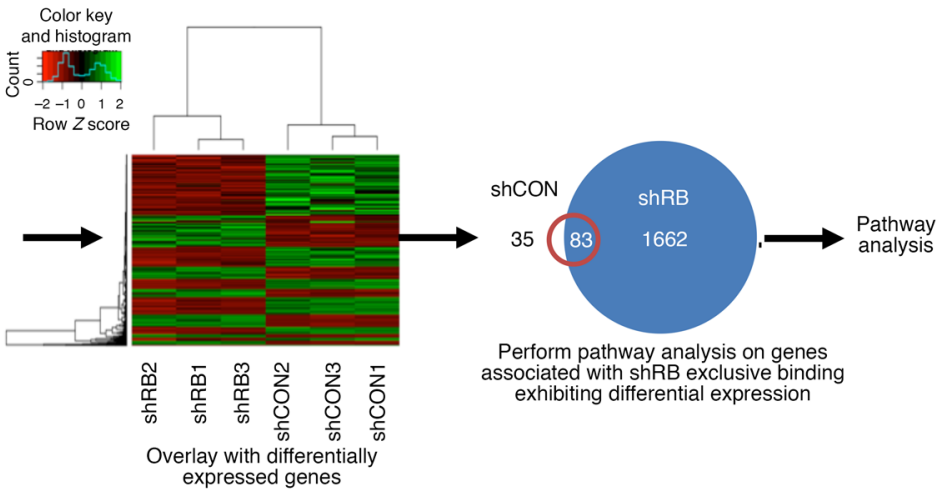

Enriched

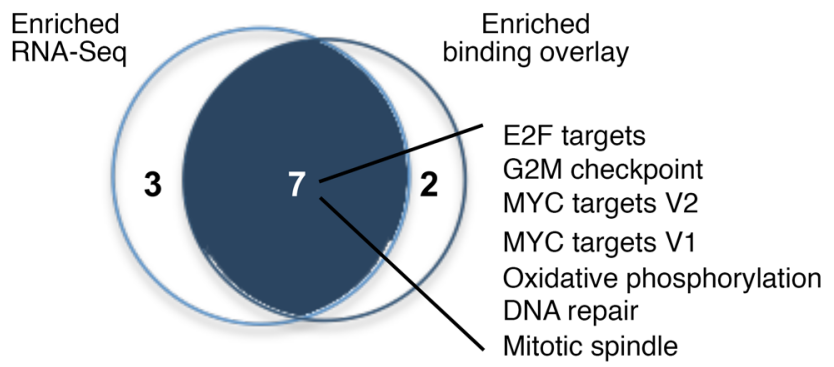


Figure 6. Genome-wide assessment of RB loss reveals novel functions and distinct transcriptional profiles. (A) Smear plot for RNA-Seq in castrate conditions (shRB vs. shCON) using Empirical Analysis of Digital Gene Expression Data in R (edgeR) to define differentially expressed genes. Genes highlighted in red represent statistically significant differential expression (adjusted $P$ value $<0.05$ ). CPM, counts per million reads mapped (B) Smear plot for RNA-Seq in shRB models (castrate vs. DHT-stimulated conditions) using edgeR to define differentially expressed genes. Genes highlighted in red represent statistically significant differential expression (adjusted $P$ value $<0.05$ ). (C) GSEA for enriched pathways upon RB loss (normalized enrichment score [NES] > 1.3). Briefly, transcriptional data from RNA-Seq output were examined for overrepresented pathways using the Hallmarks gene set collection (Molecular Signatures Database, MSigDB). Pathways de-enriched (left) or enriched (middle) after RB loss are shown, and leading-edge plot for E2F targets (right). (D) Schematic of binding to gene association with overlay with gene expression data. (E) Intersection of pathways identified from RNA-Seq alone compared with those identified using the intersection of RNA-Seq and ChIP-Seq results (NES > 1.3).

To identify pathways that were deregulated upon RB loss, gene set enrichment analysis (GSEA) was performed, using hallmark gene sets from the Molecular Signatures Database (MSigDB). Few pathways were attenuated after RB depletion, despite the total number of genes altered in this category, underscoring the focused nature of E2F1 activity to elicit specific transcriptional programs. Pathways with attenuated enrichment include those that have been previously shown to interplay with the RB/E2F1 axis, such as epithelial-mesenchymal transition, hedgehog signaling, and myogenesis (Figure 6C, left; normalized enrichment score $<-1.3$ ) (59-64). Conversely, a number of clinically relevant pathways were enriched upon RB loss, including canonical E2F targets, Myc targets, mitotic spindle, and DNA repair (Figure 6C, right; normalized enrichment score $>1.3$ ). The observed induction of E2F targets upon $\mathrm{RB}$ depletion was especially striking given the absence of mitogenic stimuli typically required to promote transcription of these targets. Similarly, the increased transcriptional output for genes responsible for DNA repair suggests that putative gained functions of E2F1 upon RB depletion may drive dysregulation of genes controlling DNA damage response, even in the absence of DNA damage. Further, Myc has been shown in other tumor types to interact with E2F3 to bring about specific transcriptional programs important for cancer progression (65). Here, transcriptional data provide some of the first evidence for RB loss-driven induction of Myc targets in prostate cancer, validated in multiple model systems, suggesting that E2F1 rather than E2F3 may interact with Myc in this disease type (Supplemental Figure 6A).

Further, to test for concordance with previously described signatures of RB loss of function, GSEA was performed using a previously described "RB loss signature" (66). As shown in Supplemental Figure 6C, genes in the RB loss signature were highly enriched within $\mathrm{RB}$ loss-induced transcriptional alterations seen in the current study, suggesting that RB loss in the current study encompasses previously described gene expression changes after functional RB inactivation. Finally, as previous studies have shown AR targets to be impacted by RB loss (36), a signature of androgen response generated from patient tissue was assessed for enrichment in the current model (67). Consistent with the previously described role for RB loss to alter AR signaling, the RB loss-induced transcriptome was significantly enriched for this gene signature (Supplemental Figure 6D). Taken together, the transcriptional changes seen upon RB depletion demonstrate gained E2F1 activity in the absence of canonically required stimuli to elicit transcriptional profiles known to be vital for aggressive disease.

To determine the potential mechanism driving the transcriptional changes observed after RB depletion, genes associated with changes in E2F1 binding after RB loss were assessed using a guilty-by-association approach (68). Briefly, to identify genes potentially regulated by novel binding, as the majority of gained binding was seen away from promoter regions, a 30-kb window around gene transcriptional start sites was used, with genes within this window deemed putatively regulated by E2F1 binding exclusive to each condition (shCON castrate, shRB castrate; Figure 6D, left). Using this approach, 317 genes showed exclusive E2F1 binding within this window with RB intact, while 599 genes harbored a gained E2F1 site in both RB-intact and RB-depleted conditions, and 12,269 genes were associated with a gained E2F1 binding site exclusive to the RB loss condition (Figure 6D, middle). Next, genes with binding exclusive to RB loss (12,269 genes) were overlaid with those exhibiting differential expression from the RNA-Seq previously described in Figure 6A to determine whether the novel E2F1 binding seen after RB loss was associated with the altered transcriptional profiles observed after RB depletion (Figure 6D, right). To more accurately assess this, GSEA was performed using the genes exhibiting gained E2F1 binding accompanied by changes in gene expression, and significantly altered pathways were compared with those identified from only transcriptional data in Figure 6C. As shown, half of the attenuated pathways identified from the RNA-Seq alone were also identified using those genes with altered expression and gained E2F1 binding (Figure 6E, left), suggesting that a subset of the pathways with reduced enrichment are likely attributable to novel E2F1 bindingmediated repression. Additionally, when pathways enriched upon RB loss were investigated, $70 \%$ of the pathways initially identified using RNA-Seq alone were recapitulated when genes with altered expression and gained E2F1 binding were analyzed (Figure $6 \mathrm{E}$, right). As the $\mathrm{RB}$ loss-driven transcriptome has heretofore remained undefined, the finding that the majority of the pathways induced after RB depletion were associated with a gain in E2F1 binding is striking, and suggests a fundamental role for the novel E2F1 cistrome identified herein as a putative driver of these pathways vital for lethal tumor phenotypes.

Evidence of expanded E2F1 activity in clinical CRPC. To assess the clinical relevance of the newly identified transcriptional networks and E2F1 repositioning driven by RB loss, genes were prioritized through iterative processes for further analyses. Briefly, genes exhibiting differential expression upon RB loss were segregated into 2 categories to determine the specific importance of gained E2F1 binding capacity clinically: (a) genes with any E2F1 binding within $30 \mathrm{~kb}$ of the transcriptional start site and (b) genes specifically associated with gained E2F1 binding after RB loss (Figure $6 \mathrm{D})$. These 2 gene sets were then queried against gene expression data from the SU2C/PCF CRPC tumor cohort. Genes exhibiting expression changes upon RB loss in the SU2C/PCF cohort concordant with our model were identified, resulting in a list of genes with 
A

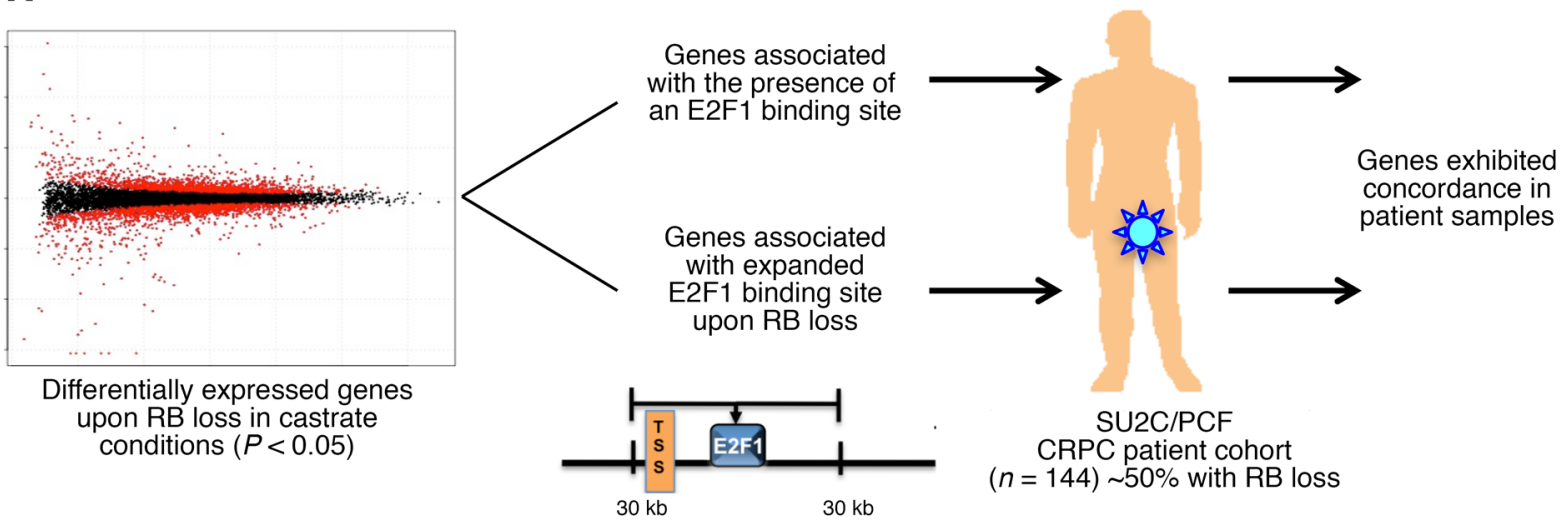

B

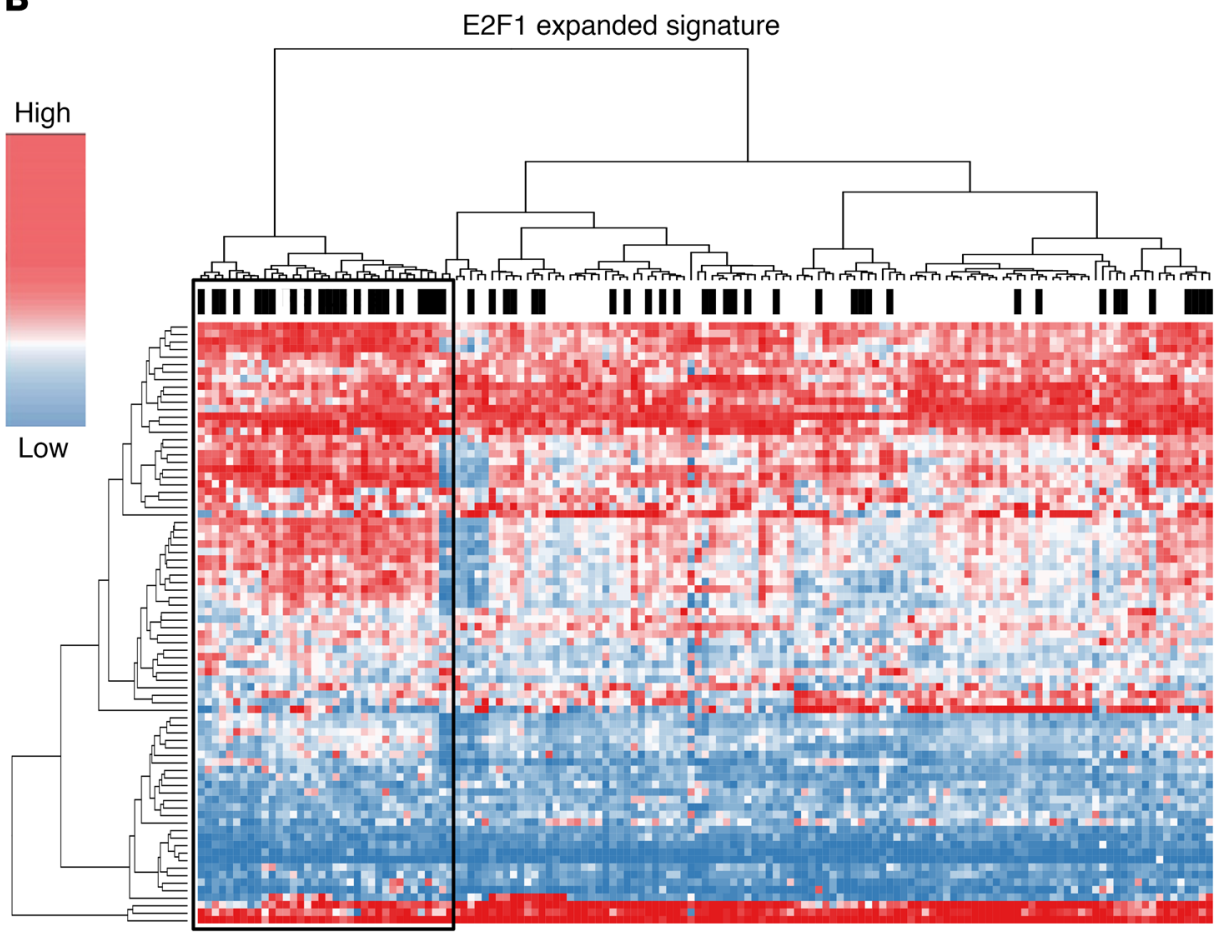

C

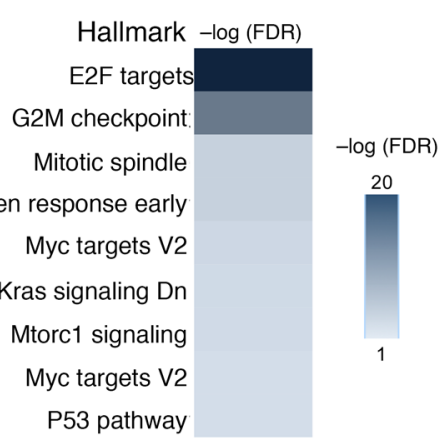

Figure 7. Clinical assessment of RB loss in CRPC reveals novel E2F1-gained cistrome-driven gene signature. (A) Differentially expressed genes from RNA-Seq were overlaid with ChIP-Seq binding studies into those genes that were associated with an E2F1 binding site, or those genes associated with a gained E2F1 binding site (seen only upon RB loss). Genes in each of these categories were then queried against CRPC patient data from the SU2C cohort, and those genes from each category with expression concordant with the initial model identified. TSS, transcriptional start site. (B) Hierarchical clustering of normalized expression data of concordant genes from 144 SU2C CRPC samples (1 - Pearson's correlation coefficient for columns and Euclidean distance for rows were used as distance measures). The annotation track reports the genomic status of RB1 (black, SCNA alteration; white, no SCNA alteration) and genes used for heatmap generation identified through analysis in A. (C) CSEA was performed on the 80-gene signature identified in B, in order to identify which pathways were likely to drive signaling in CRPC patients.

E2F1 binding (present or exclusively gained after RB loss), differential expression in models of RB loss, and differential expression in clinical patient samples after RB loss (Figure 7A).

To examine the clinical relevance of RB loss-induced E2F1 reprogramming and altered transcriptional output identified in the present study, hierarchical clustering was performed using genes identified in Figure $6 \mathrm{~A}$ and transcriptional data from the SU2C/PCF CRPC tumor cohort (Figure 7A). Importantly, genes specifically exhibiting gained E2F1 binding sites upon RB loss (deemed "E2F1 expanded signature") displayed a significant abil- ity to cluster tumors exhibiting RB loss together in the SU2C/PCF cohort $(P=0.002$, odds ratio $=3.7)$ compared with those genes with the presence of an E2F1 binding site (not significant), suggesting that genes exhibiting RB loss-induced differential expression and expanded E2F1 binding in this model better predict for $\mathrm{RB}$ loss in clinical samples than the simple presence of an E2F1 site alone (Figure 7B and Supplemental Figure 7A). As such, these findings demonstrate that the capacity of $\mathrm{RB}$ loss to reprogram E2F1 binding capacity and the resultant downstream transcriptional networks occurs in clinically lethal CRPC. Furthermore, 
interrogation of the resulting gene signature further highlights the protumorigenic consequence of RB loss that is distinct from cell cycle control. Using GSEA analyses, pathways enriched within this signature included not only E2F targets, but also networks regulating Myc function, mitotic spindle integrity, and p53 response (Figure 7, B and C). Further, to assess how the E2F1 expanded signature identified in the current study compared with "ideal" clustering for RB loss in the SU2C/PCF cohort, supervised analysis was performed using only SU2C/PCF gene expression data to identify genes significantly changed in $\mathrm{RB}$-altered versus RB wild-type samples. As shown in Supplemental Figure 7B, clustering resulting from supervised analysis was comparable to that generated using the E2F1 expanded signature. Moreover, genes contained in the E2F1 expanded signature were seen to be significantly enriched within the gene set resulting from supervised analysis by both GSEA (FDR $=0.003)$ and Fisher's exact test $\left(P<1 \times 10^{-10}\right)$ (Supplemental Figure $7 \mathrm{C}$ ), further supporting the significance of gained E2F1 binding upon RB loss within the SU2C/ PCF cohort. Together, these data demonstrate that the expanded E2F1 cistrome identified herein occurs in RB-deficient clinical CRPC, providing the first insight into the mechanisms by which $\mathrm{RB}$ loss induces aggressive tumor phenotypes, independent of cell cycle dysregulation (Figure 8).

\section{Discussion}

The importance of the RB/E2F1 pathway in tumor suppression is compellingly illustrated by the frequency of pathway disruption in human malignancy and association with poor outcome (34-36, 38, 69). However, the underlying basis for tumor-specific RB inactivation versus $\mathrm{RB}$ loss remains unknown. The present study reveals the molecular consequences of RB loss in human disease, and identifies unique molecular and cellular outcomes that promote tumor aggressiveness. Key findings include: (a) in CRPC, alterations of the RB pathway are preferentially enriched for RB loss, which is frequently observed in both tumor samples and ctDNA from patients with advanced disease (Figure 1); (b) loss of RB is associated with advanced disease but is independent of altered proliferative indices, suggesting roles for RB outside cell cycle control (Figure 2); (c) RB loss expands and reprograms E2F1 chromatin occupancy (Figure 3); (d) the molecular consequence of RB loss is distinct from that observed after RB inactivation (Figures 3 and 4); (e) gained E2F1 binding capacity is not likely to be the result of widespread, global changes in chromatin accessibility (Figure 5); (f) $\mathrm{RB}$ depletion induces distinct transcriptional networks that are highly associated with gained E2F1 activity after RB loss (Figure 6); and (g) the identified transcriptional programs driven by the gained activity of E2F1 upon RB loss ("E2F1 expanded signature") occur in clinical, end-stage disease (Figure 7). Taken together, these findings define the molecular and tumor-associated consequences of RB loss in human malignancy, and provide the first insight into the putative mechanism of RB loss-induced transcriptional alterations in advanced cancers.

As demonstrated in both preclinical modeling and clinical samples, it is clear that reprogrammed E2F1 activity after RB loss elicits biological effects distinct from cell cycle control. As described herein, analyses of 2 large, independent CRPC cohorts indicate that loss of $\mathrm{RB}$ is the main mechanism of $\mathrm{RB}$ pathway disruption in this malignancy. While additional studies will be used to assess the potential for altered AR activity to impact RB phosphorylation status in late-stage disease, data in the present study reveal no correlation between loss of $\mathrm{RB}$ and increased Ki67 in CRPC, demonstrating that RB loss is not sufficient to alter proliferation and further suggesting that the capacity of RB depletion to drive aggressive tumor phenotypes occurs via functions distinct from cell cycle control (Figure 2, $\mathrm{B}$ and $\mathrm{C}$ ). While investigation of associations between RB pathway status and Ki67 in the clinical setting is limited, the concept that RB loss induces biological effects distinct from cell cycle deregulation has precedent in human tumors. For instance, in a recent study of lung adenocarcinoma (70), RB-negative tumors were seen to have no correlation with Ki67 positivity $(P=0.33)$, further supporting findings in the current study that suggest $\mathrm{RB}$ loss is not associated with hyperproliferative phenotypes (Figure 2, B and C). Conversely, in other tumor types where $\mathrm{RB}$ expression is retained but inactivated by phosphorylation, correlation with a higher proliferative index was observed, including studies in diffuse large B cell lymphoma, in Burkitt's lymphoma wherein $\mathrm{p}^{27^{\mathrm{Kip} 1}}$ is lost $(71,72)$, or in luminal A and triple-negative breast cancer where p16 ${ }^{\mathrm{INK} 4 \mathrm{~A}}$ loss occurs (21). In concert with the data herein, these findings shift thinking with regard to $\mathrm{RB}$ pathway disruption, and provide compelling clinical evidence to support the contention that RB loss is mechanistically distinct from $\mathrm{RB}$ inactivation.

The discovery that RB loss elicits distinct effects compared with $\mathrm{RB}$ functional inactivation in clinical tumor samples is striking, as RB loss alone is sufficient to induce CRPC phenotypes (36) and loss of RB was seen to be the main mechanism of RB pathway disruption in the current study. Further, as models of RB loss are reliant on E2F1 for castration-resistant growth (36), provision of a putative mechanism for E2F1 in mediating these downstream phenotypes is significant. Toward this end, a key discovery herein demonstrated, through use of multiple model systems, is that the E2F1 cistrome is differentially expanded after RB loss, suggesting that E2F1 binding is not in fact a static phenomenon. Further, studies presented here demonstrate, for the first time to our knowledge, that RB loss (as compared with RB inactivation) significantly expands the putative repertoire of E2F1-regulated target genes through binding outside of promoter regions, with $16,292 \mathrm{E} 2 \mathrm{~F} 1$ sites gained after RB loss, $31.2 \%$ and $32.6 \%$ of binding occurring in intronic and intergenic regions, respectively (Figure 3, A and B). As E2F1 was previously thought to regulate target genes through promoter binding (2), these findings open new avenues of understanding for discerning the function(s) of E2F1 in promoting disease progression when $\mathrm{RB}$ is lost. Moreover, as discerning the molecular basis of expanded E2F1 binding is of interest, it is striking that chromatin accessibility was largely unchanged regardless of RB status, suggesting that the mechanisms underlying the altered E2F1 cistrome lie outside simple opportunistic binding due to changes in open chromatin (Figure 5). Thus, current investigation is focused on discerning the molecular basis of expanded E2F1 binding upon RB loss.

Data herein further implicate gained E2F1 chromatin localization outside canonical promoter regions, through potential use of a noncanonical minimum-affinity binding motif previ- 
A

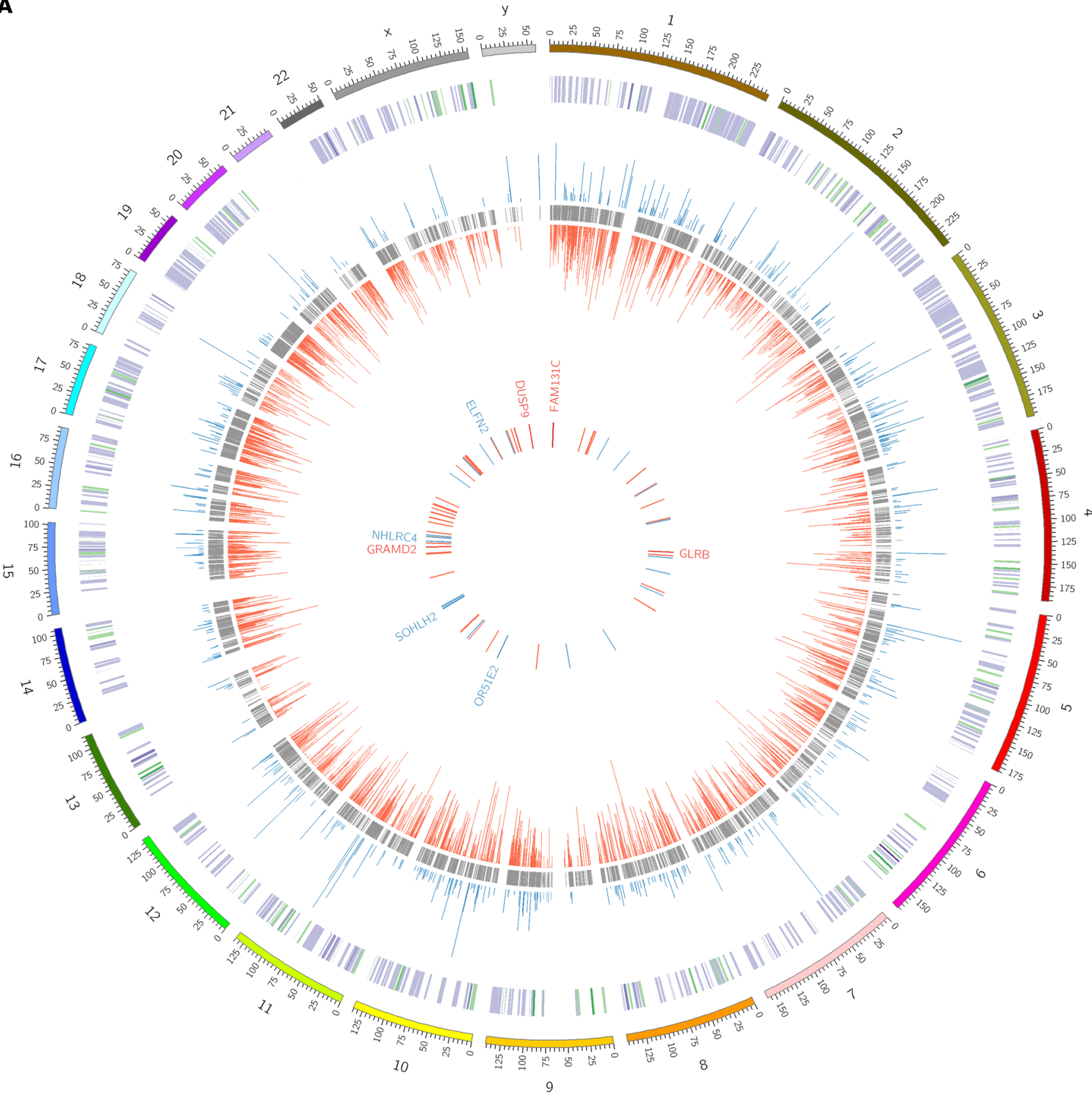

B
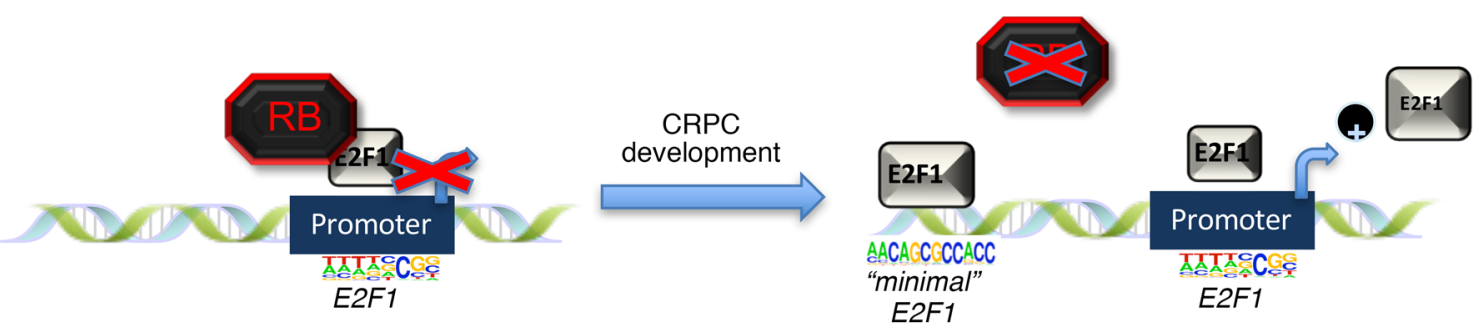
Figure 8. Summary of E2F1-induced alterations upon RB loss in CRPC. (A) Circos plot represents ChIP-Seq, RNA-Seq, and clinical alterations identified in the current study. From outer ring to inner ring, the levels represent: (i) differentially expressed genes identified through RNA-Seq after RB loss (purple/green heatmap), (ii) E2F1 binding exclusive to shCON condition (blue histogram), (iii) binding common to shCON and shRB conditions (gray), (iv) E2F1 binding exclusive to shRB condition (red histogram), and (v) genes exhibiting differential expression after RB loss, associated with gained E2F1 binding, and concordant with SU2C clinical expression data. (B) Hypothesized model of RB loss in CRPC.

ously identified in studies assessing E2F1 functional domains in addition to those investigating E2F1 function in liver cancer (refs. 46, 48, and Figure 3, C and D). Interestingly, this abbreviated, minimum-affinity E2F motif was found to be enriched in regions of AR binding as well as under epigenetic marks of active transcription (H3K4me3) in recent studies in CRPC tissue (67), suggesting that E2F1 may preferentially interact with AR in CRPC through gained binding capacity at minimum-affinity regions. Moreover, motifs for binding of putative cooperating transcription factors of clinical relevance were enriched in conjunction with gained E2F1 occupancy after RB loss (Figure 3D), including those associated with: NF1 factors, which can modulate AR function $(73,74)$; TAL-1 (SCL), which influences pioneer factors that modulate AR function $(75,76)$; and the half-site for AR itself. Further, de novo motif analyses additionally implicate AR, as the RB loss-induced E2F1 cistrome was enriched for an AR half-site (Figure 4C) (77). These observations suggest the intriguing concept, currently being tested, that E2F1 reprogramming may influence AR activity, or vice versa. Finally, gained E2F1 binding is associated with enrichment for the orphan nuclear receptor TLX, which promotes prostate cancer growth and is highly expressed in advanced disease (78). Combined, these data indicate that loss of RB expands E2F1 activity in a manner distinct from $\mathrm{RB}$ retention/inactivation, allowing E2F1 to localize to new, distal genomic sites governed distinctly from promoter regions (Figures 3 and 4).

Consistent with the molecular identification of the differential RB-mediated E2F1 cistrome, a distinct transcriptome was defined, which was driven specifically by RB depletion and highly associated with E2F1 reprogramming in the context of RB loss (Figure 5). Discovery of transcriptional networks that are uniquely associated with expanded E2F1 occupancy underscored activities of E2F1 beyond those linked to cell cycle control (Figure 6, $\mathrm{C}$ and $\mathrm{E}$ ), and identified heretofore underappreciated functions of E2F1. Notably, E2F1 reprogramming appears to be associated with increased Myc target gene expression, which is known to promote disease progression and poor outcome in this tumor type $(79,80)$. These findings may also provide a molecular basis for recent observations in intestinal cancers, wherein RB pathway loss induces E2F3 and Myc cooperation (65). As such, while E2F3 does not significantly correlate with RB loss in CRPC (36), the potential for effects on the RB loss-induced E2F3 cistrome represents an intriguing node of future study. In addition to associations with Myc function, it is striking that E2F1 reprogramming enriches pathways promoting DNA damage and repair, given recent findings linking DNA repair alterations with disease progression and acquisition of aggressive tumor phenotypes (81-86).
Together, these findings bring about broad new understanding of the biological impact of RB loss on tumor phenotypes that promote progression; ongoing studies are directed at discerning the relative contribution of each newly identified E2F1-associated network to biological outcomes and disease progression.

Finally, findings in the current study identify a signature of genes predictive for RB loss in CRPC, associated with gained E2F1 binding in the model systems used in the current study (Figure 7). Remarkably, only genes exclusively associated with expanded E2F1 binding driven by RB loss clustered CRPC tumors deficient in RB expression, while the simple presence of $\mathrm{E} 2 \mathrm{~F} 1$ was unable to predict for the same alteration in the tumor samples, suggesting that $\mathrm{E} 2 \mathrm{~F} 1$ binding gained after RB loss is significantly relevant to CRPC phenotypes (Figure 7B and Supplemental Figure 7). These data identify, for the first time, a set of targets induced through novel E2F1 binding, and specifically altered by RB loss in human disease. Importantly, higher stringency used to define differentially expressed genes in the model systems used herein identified stronger concordance between in vitro expression data and gene expression profiles from patient tumor samples, further highlighting the clinical relevance of the targets identified in these models. It is of note that the current study used models of AR-positive disease in addition to non-neuroendocrine CRPC tumor samples (SU2C/PCF), as recent studies suggest that RB loss may yield distinct effects in tumors acquiring neuroendocrine phenotypes via RB loss $(39,87)$. Further, as RB loss of expression has been shown in the current study to elicit molecular effects distinct from those driven by phosphorylation-induced inactivation, it is likely that this RB loss-induced gene signature (E2F1 expanded signature) could be applied to other disease types to indicate similar E2F1 reprogramming. Additionally, pathway analyses for genes within this signature were highly consistent with those identified using binding and gene expression in the model systems alone, suggesting a coordinated set of pathways preferentially driven by E2F1 binding both in vitro and in advanced disease (Figure 6E and Figure 7C). Thus, the identification of specific RB loss-altered gene targets within pathways that drive CRPC phenotypes represents potential targets for further therapeutic intervention.

Taken together, molecular interrogation of E2F1 function after RB loss suggests a paradigm shift, whereby E2F1 binding is directed away from promoter regions, eliciting marked reprogramming of E2F1 chromatin-binding capacity and E2F1associated transcriptional programs. Further, data herein coupled with clinical studies indicate that E2F1 activity after RB loss is distinct from E2F1 activity after phosphorylation-induced RB inactivation, suggesting a distinction in how RB protein depletion and loss of function through inactivation impact human malignancy. Finally, data in the current study nominate a novel gene signature driven by RB loss and gained E2F1 function, which is altered in late-stage prostate cancer and is predictive for RB loss in advanced disease ("E2F1 expanded signature"), further nominating E2F1 as a critical driver of CRPC phenotypes in this disease state. Together, these findings contribute new insights into the molecular mechanisms underpinning RB lossdependent CRPC phenotypes, and define a novel E2F1 cistrome upon RB loss associated with an altered transcriptional program with applicability to multiple malignancies. 


\section{Methods}

ChIP sequencing. ChIP was performed as described previously (88). Briefly, $8 \times 10^{6}$ to $10 \times 10^{6}$ cells were cross-linked with $1 \%$ formaldehyde for 10 minutes at room temperature. Nuclei were extracted using LB3 buffer (10 mM Tris-HCl [pH 8.0], $100 \mathrm{mM} \mathrm{NaCl}, 1 \mathrm{mM}$ EDTA [pH 8.0], 0.5 mM EGTA [pH 8.0], 0.1\% Na-deoxycholate, $0.5 \% \mathrm{~N}$ lauroylsarcosine), and chromatin was sheared to 200-700 bp using Covaris E220 Focused Ultrasonicator (Covaris). E2F1 antibodies for ChIP assays were purchased from BD Pharmingen (catalog 554213) and Santa Cruz Biotechnology (sc-193). The ChIP-Seq libraries were constructed using the ThruPLEX-FD Prep Kit (Rubicon Genomics) with approximately $10 \mathrm{ng}$ of ChIP DNA. Final products were sequenced on the NextSeq 500 at the Molecular Biology Core Facilities of Dana-Farber Cancer Institute. ChIP-Seq data have been deposited in the GEO repository under the accession number GSE94958.

RNA sequencing. RNA-Seq samples were prepared as described previously (47). Briefly, total RNAs were extracted and purified using the TRIzol reagent and RNeasy Mini Kit (Qiagen) according to the manufacturer's instructions. RNA-Seq libraries were constructed using TruSeq RNA Sample Prep Kit v2 (Illumina, RS-122-2001) and then sequenced on the NextSeq 500 at the Molecular Biology Core Facilities of DanaFarber Cancer Institute. RNA-Seq data have been deposited in the GEO repository under the accession number GSE94863.

ATAC-Seq. ATAC-Seq was performed as previously described (89). Briefly, cells were plated and treated as indicated by the condition. Next, 75,000 cells were collected and lysed in ATAC lysis buffer. Cells were then resuspended in the transposition reaction mix, and the transposition reaction was incubated at $37^{\circ} \mathrm{C}$ for 30 minutes. Transposed DNA was purified using AMPure purification methods as specified by the manufacturer (Agencourt). Purified DNA was then amplified using custom barcoded Nextera primers as previously reported and available in Buenrostro et al. (89). Library size and quality were validated using a Bioanalyzer and libraries sequenced on a NextSeq 500. ATAC-Seq data have been deposited in the GEO repository under the accession number GSE105116.

Androgen stimulation. In androgen stimulation conditions, cells were cultured in $5 \%$ charcoal dextran-treated (CDT-treated) media for 72 hours before treatment with $10 \mathrm{~nm}$ DHT or vehicle (EtOH) for 3 hours prior to harvest.

Quantitative PCR. Quantitative PCR (qPCR) analysis for occupancy was performed as previously described for both ChIP-qPCR and reverse transcription qPCR $(47,88)$.

Immunoblot analysis. Cells were plated in CDT for 72 hours before harvest. Cell lysates were generated and immunoblot performed as previously described (68). Antibodies used were as follows: RB (BD Pharmingen, 554136), E2F1 (Santa Cruz Biotechnology, sc-193), GAPDH (Santa Cruz Biotechnology, sc-25778), p780-RB (Cell Signaling Technology, 3590S).

Statistics. Statistical analyses were performed as described above. Briefly, IHC correlations were calculated by Spearman correlation, with a $P$ less than 0.05 considered significant. Significance for ChIPSeq and differential gene expression for RNA-Seq were calculated by the indicated software as described above. Motif analysis significance was calculated by the Homer suite, with a $P$ less than $1 \times 10^{-20}$ considered significant. Concordance with the study model and SU2C/PCF cohort was calculated as described above. Individual thresholds for significance are additionally indicated in applicable figure legends. Data are expressed as mean \pm SD unless otherwise indicated.

Study approval. Use of patient and clinical material was approved by the ethical committee of Tampere University Hospital and the National Authority for Medicolegal Affairs (Helsinki, Finland), by the Marsden Ethics Committee (London, United Kingdom, CCR-2472), by the regional ethical vetting board of Stockholm, Sweden (register number 2009/1357-32 and amendment registry number 2014/156432 ), and by the institutional review and ethics board of GZA SintAugustinus (Wilrijk, Belgium). Informed consent was obtained in all cases excluding samples from the Tampere cohort, where, according to Finnish law, in cases in which informed consent cannot be obtained as a result of studies involving large retrospective materials or patients who have died before study, permission can be given by the National Authority for Medicolegal Affairs.

Further information can be found in Supplemental Methods, available online with this article; https://doi.org/10.1172/JCI93566DS1.

\section{Author contributions}

CM, M Brown, MAR, JdeB, FD, FYF, TV, and KEK designed the studies. CM, ACM, and KX performed experiments. CM, M Benelli, BL, DR, JL, HG, MC, BDL, LD, TV, FL, and FD acquired and/or analyzed data for the study. CM and KEK wrote the manuscript along with input from all authors.

\section{Acknowledgments}

We gratefully thank all members of the Knudsen laboratory for their invaluable input and support. We also thank the institutions that supported this work: the National Cancer Institute (CA159945, CA217329, and CA176401 to KEK, 4R00CA178199 to $\mathrm{KX}$ ), the Cancer Prevention Research Institute of Texas (RR140072 to KX), the Sidney Kimmel Cancer Center (Support Grant 5P30CA056036-17 to KEK) and Biostatistics Shared Resource (to $\mathrm{BL}$ ), the Prostate Cancer Foundation (Challenge Award to KEK, JdeB, MAR, FYF), the European Research Council (ERCCoG648670 to FD), and, in part, the Pennsylvania Department of Health (grant to KEK). The Department specifically disclaims responsibility for any analyses, interpretations, or conclusions.

Address correspondence to: Karen E. Knudsen, Sidney Kimmel Cancer Center, 233 South 10th Street, BLSB 1050, Philadelphia, Pennsylvania 19107, USA. Phone: 215.503.7595; E-mail: Karen. knudsen@jefferson.edu.
1. Dyson NJ. RB1: a prototype tumor suppressor and an enigma. Genes Dev. 2016;30(13):1492-1502.

2. van den Heuvel S, Dyson NJ. Conserved functions of the $\mathrm{pRB}$ and E2F families. Nat Rev Mol Cell Biol. 2008;9(9):713-724.

3. Chen HZ, Tsai SY, Leone G. Emerging roles of
E2Fs in cancer: an exit from cell cycle control. Nat Rev Cancer. 2009;9(11):785-797.

4. Trimarchi JM, Lees JA. Sibling rivalry in the E2F family. Nat Rev Mol Cell Biol. 2002;3(1):11-20.

5. Biswas AK, Johnson DG. Transcriptional and nontranscriptional functions of E2F1 in response to DNA damage. Cancer Res. 2012;72(1):13-17.

6. Chen $\mathrm{H}$, et al. PDGF signalling controls agedependent proliferation in pancreatic $\beta$-cells. Nature. 2011;478(7369):349-355.

7. Biswas AK, Mitchell DL, Johnson DG. E2F1 responds to ultraviolet radiation by directly 
stimulating DNA repair and suppressing carcinogenesis. Cancer Res. 2014;74(12):3369-3377.

8. Burkhart DL, Sage J. Cellular mechanisms of tumour suppression by the retinoblastoma gene. Nat Rev Cancer. 2008;8(9):671-682.

9. Sherr CJ. Cancer cell cycles. Science. 1996;274(5293):1672-1677.

10. Attwooll C, et al. A novel repressive E2F6 complex containing the polycomb group protein, EPC1, that interacts with $\mathrm{EZH} 2$ in a proliferation-specific manner. J Biol Chem. 2005;280(2):1199-1208.

11. Asghar U, Witkiewicz AK, Turner NC, Knudsen ES. The history and future of targeting cyclindependent kinases in cancer therapy. Nat Rev Drug Discov. 2015;14(2):130-146.

12. Khatib ZA, Matsushime H, Valentine M, Shapiro DN, Sherr CJ, Look AT. Coamplification of the CDK4 gene with MDM2 and GLI in human sarcomas. Cancer Res. 1993;53(22):5535-5541.

13. Musgrove EA, Caldon CE, Barraclough J, Stone A, Sutherland RL. Cyclin D as a therapeutic target in cancer. Nat Rev Cancer. 2011;11(8):558-572.

14. Park S, et al. Aberrant CDK4 amplification in refractory rhabdomyosarcoma as identified by genomic profiling. Sci Rep. 2014;4:3623.

15. Knudsen ES, Knudsen KE. Tailoring to RB: tumour suppressor status and therapeutic response. Nat Rev Cancer. 2008;8(9):714-724.

16. Sherr CJ, Beach D, Shapiro GI. Targeting CDK4 and CDK6: from discovery to therapy. Cancer Discov. 2016;6(4):353-367.

17. Bartoletti R, Cai T, Nesi G, Roberta Girardi L, Baroni G, Dal Canto M. Loss of P16 expression and chromosome 9p21 LOH in predicting outcome of patients affected by superficial bladder cancer. J Surg Res. 2007;143(2):422-427.

18. Dreyling MH, et al. Alterations of the cyclin D1/ p16-pRB pathway in mantle cell lymphoma. Cancer Res. 1997;57(20):4608-4614.

19. Gerdes B, et al. p16INK4a is a prognostic marker in resected ductal pancreatic cancer: an analysis of p16INK4a, p53, MDM2, an Rb. Ann Surg. 2002;235(1):51-59.

20. LaPak KM, Burd CE. The molecular balancing act of p16(INK4a) in cancer and aging. Mol Cancer Res. 2014;12(2):167-183.

21. Shin E, Jung WH, Koo JS. Expression of p16 and pRB in invasive breast cancer. Int J Clin Exp Pathol. 2015;8(7):8209-8217.

22. Straume O, Sviland L, Akslen LA. Loss of nuclear p16 protein expression correlates with increased tumor cell proliferation (Ki-67) and poor prognosis in patients with vertical growth phase melanoma. Clin Cancer Res. 2000;6(5):1845-1853.

23. Zhao R, Choi BY, Lee MH, Bode AM, Dong Z. Implications of genetic and epigenetic alterations of CDKN2A (p16(INK4a)) in cancer. EBioMedicine. 2016;8:30-39.

24. Comstock CE, et al. Targeting cell cycle and hormone receptor pathways in cancer. Oncogene. 2013;32(48):5481-5491.

25. Finn RS, et al. PD 0332991, a selective cyclin D kinase $4 / 6$ inhibitor, preferentially inhibits proliferation of luminal estrogen receptor-positive human breast cancer cell lines in vitro. Breast Cancer Res. 2009;11(5):R77.

26. Finn RS, et al. The cyclin-dependent kinase $4 / 6$ inhibitor palbociclib in combination with letro- zole versus letrozole alone as first-line treatment of oestrogen receptor-positive, HER2negative, advanced breast cancer (PALOMA-1/ TRIO-18): a randomised phase 2 study. Lancet Oncol. 2015;16(1):25-35.

27. Palbociclib. US Food and Drug Administration. FDA Website https://www.fda.gov/drugs/ informationondrugs/approveddrugs/ ucm549978.htm. Updated March 31, 2017. Accessed November 2, 2017.

28. Di Fiore R, D'Anneo A, Tesoriere G, Vento R. RB1 in cancer: different mechanisms of RB1 inactivation and alterations of $\mathrm{pRb}$ pathway in tumorigenesis. JCell Physiol. 2013;228(8):1676-1687.

29. Giacinti C, Giordano A. RB and cell cycle progression. Oncogene. 2006;25(38):5220-5227.

30. Viatour P, Sage J. Newly identified aspects of tumor suppression by RB. Dis Model Mech . 2011;4(5):581-585.

31. Niederst MJ, et al. RB loss in resistant EGFR mutant lung adenocarcinomas that transform to small-cell lung cancer. Nat Commun. 2015;6:6377.

32. Oser MG, Niederst MJ, Sequist LV, Engelman JA. Transformation from non-small-cell lung cancer to small-cell lung cancer: molecular drivers and cells of origin. Lancet Oncol. 2015;16(4):e165-e172.

33. Robinson $\mathrm{D}$, et al. Integrative clinical genomics of advanced prostate cancer. Cell. 2015;161(5):1215-1228.

34 . Taylor BS, et al. Integrative genomic profiling of human prostate cancer. Cancer Cell. 2010;18(1):11-22.

35. Aparicio A, Den RB, Knudsen KE. Time to stratify? The retinoblastoma protein in castrate-resistant prostate cancer. Nat Rev Urol. 2011;8(10):562-568.

36. Sharma A, et al. The retinoblastoma tumor suppressor controls androgen signaling and human prostate cancer progression. J Clin Invest. 2010;120(12):4478-4492.

37. de Leeuw R, et al. Novel actions of nextgeneration taxanes benefit advanced stages of prostate cancer. Clin Cancer Res. 2015;21(4):795-807.

38. Sharma A, et al. Retinoblastoma tumor suppressor status is a critical determinant of therapeutic response in prostate cancer cells. Cancer Res. 2007;67(13):6192-6203.

39. $\mathrm{Mu}$ P, et al. SOX 2 promotes lineage plasticity and antiandrogen resistance in TP53- and RB1-deficient prostate cancer. Science. 2017;355(6320):84-88.

40. Knudsen KE, Penning TM. Partners in crime: deregulation of AR activity and androgen synthesis in prostate cancer. Trends Endocrinol Metab. 2010;21(5):315-324.

41. Boyd LK, Mao X, Lu YJ. The complexity of prostate cancer: genomic alterations and heterogeneity. Nat Rev Urol. 2012;9(11):652-664.

42. Beltran $\mathrm{H}$, et al. Divergent clonal evolution of castration-resistant neuroendocrine prostate cancer. Nat Med. 2016;22(3):298-305.

43. Berman-Booty LD, Knudsen KE. Models of neuroendocrine prostate cancer. Endocr Relat Cancer. 2015;22(1):R33-R49.

44. Watson PA, Arora VK, Sawyers CL. Emerging mechanisms of resistance to androgen receptor inhibitors in prostate cancer. Nat Rev Cancer. 2015;15(12):701-711.

45. Ramos-Montoya A, et al. HES6 drives a critical AR transcriptional programme to induce castrationresistant prostate cancer through activation of an E2F1-mediated cell cycle network. EMBO Mol Med. 2014;6(5):651-661.

46. Cao AR, Rabinovich R, Xu M, Xu X, Jin VX, Farnham PJ. Genome-wide analysis of transcription factor E2F1 mutant proteins reveals that $\mathrm{N}$ - and $\mathrm{C}$-terminal protein interaction domains do not participate in targeting E2F1 to the human genome. J Biol Chem. 2011;286(14):11985-11996.

47. Xu H, et al. Integrative analysis reveals the transcriptional collaboration between EZH2 and E2F1 in the regulation of cancer-related gene expression. Mol Cancer Res. 2016;14(2):163-172.

48. Tarangelo A, et al. Recruitment of Pontin/Reptin by E2f1 amplifies E2f transcriptional response during cancer progression. Nat Commun. 2015;6:10028.

49. Xu Y, Chen SY, Ross KN, Balk SP. Androgens induce prostate cancer cell proliferation through mammalian target of rapamycin activation and post-transcriptional increases in cyclin $D$ proteins. Cancer Res. 2006;66(15):7783-7792.

50. Gao S, et al. Androgen receptor tumor suppressor function is mediated by recruitment of retinoblastoma protein. Cell Rep. 2016;17(4):966-976.

51. Altucci L, et al. 17beta-Estradiol induces cyclin D1 gene transcription, p36D1-p34cdk4 complex activation and $\mathrm{p} 105 \mathrm{Rb}$ phosphorylation during mitogenic stimulation of $\mathrm{G}(1)$-arrested human breast cancer cells. Oncogene. 1996;12(11):2315-2324.

52. Foster JS, Wimalasena J. Estrogen regulates activity of cyclin-dependent kinases and retinoblastoma protein phosphorylation in breast cancer cells. Mol Endocrinol. 1996;10(5):488-498.

53. Prall OW, Sarcevic B, Musgrove EA, Watts CK, Sutherland RL. Estrogen-induced activation of Cdk4 and Cdk2 during G1-S phase progression is accompanied by increased cyclin D1 expression and decreased cyclin-dependent kinase inhibitor association with cyclin E-Cdk2.J Biol Chem. 1997;272(16):10882-10894.

54. Eeckhoute J, Carroll JS, Geistlinger TR, Torres-Arzayus MI, Brown M. A cell-typespecific transcriptional network required for estrogen regulation of cyclin D1 and cell cycle progression in breast cancer. Genes Dev. 2006;20 (18):2513-2526.

55. Andrusiak MG, Vandenbosch R, Dick FA, Park DS, Slack RS. LXCXE-independent chromatin remodeling by $\mathrm{Rb} / \mathrm{E} 2 \mathrm{f}$ mediates neuronal quiescence. Cell Cycle. 2013;12(9):1416-1423.

56. Brehm A, Miska EA, McCance DJ, Reid JL, Bannister AJ, Kouzarides T. Retinoblastoma protein recruits histone deacetylase to repress transcription. Nature. 1998;391(6667):597-601.

57. Magnaghi-Jaulin L, et al. Retinoblastoma protein represses transcription by recruiting a histone deacetylase. Nature. 1998;391(6667):601-605.

58. Talluri S, Dick FA. Regulation of transcription and chromatin structure by pRB: here, there and everywhere. Cell Cycle. 2012;11(17):3189-3198.

59. Knoll S, et al. E2F1 induces miR-224/452 expression to drive EMT through TXNIP downregulation. EMBO Rep. 2014;15(12):1315-1329. 
60. Guo K, Walsh K. Inhibition of myogenesis by multiple cyclin-Cdk complexes. Coordinate regulation of myogenesis and cell cycle activity at the level of E2F. J Biol Chem. 1997;272(2):791-797.

61. Ma X, et al. Overexpression of E2F1 promotes tumor malignancy and correlates with TNM stages in clear cell renal cell carcinoma. PLoS One. 2013;8(9):e73436.

62. Pandolfi S, Montagnani V, Lapucci A, Stecca B. HEDGEHOG/GLI-E2F1 axis modulates iASPP expression and function and regulates melanoma cell growth. Cell Death Differ. 2015;22(12):2006-2019.

63. Wang J, Helin K, Jin P, Nadal-Ginard B. Inhibition of in vitro myogenic differentiation by cellular transcription factor E2F1. Cell Growth Differ. 1995;6(10):1299-1306.

64. Thangavel C, et al. RB loss promotes prostate cancer metastasis. Cancer Res. 2017;77(4):982-995.

65. Liu H, et al. Redeployment of Myc and E2f1-3 drives Rb-deficient cell cycles. Nat Cell Biol. 2015;17(8):1036-1048.

66. Ertel A, et al. RB-pathway disruption in breast cancer: differential association with disease subtypes, disease-specific prognosis and therapeutic response. Cell Cycle. 2010;9(20):4153-4163.

67. Sharma NL, et al. The androgen receptor induces a distinct transcriptional program in castrationresistant prostate cancer in man. Cancer Cell. 2013;23(1):35-47.

68. McNair C, et al. Cell cycle-coupled expansion of AR activity promotes cancer progression. Oncogene. 2017;36(12):1655-1668.

69. Beltran H, et al. Targeted next-generation sequencing of advanced prostate cancer identifies potential therapeutic targets and disease heterogeneity. Eur Urol. 2013;63(5):920-926.

70. Cecchini MJ, et al. Loss of the retinoblastoma tumor suppressor correlates with improved outcome in patients with lung adenocarcinoma treated with surgery and chemotherapy. Hum Pathol. 2015;46(12):1922-1934.

71. Sánchez-Beato M, et al. Cyclin-dependent kinase inhibitor p27KIP1 in lymphoid tissue: p27KIP1 expression is inversely proportional to the proliferative index. Am J Pathol. 1997;151(1):151-160.

72. Kiviniemi M, Sauroja I, Rajamäki A, Punnonen K, Söderström KO, Salminen E. Cell cycle regulators $\mathrm{p} 27$ and pRb in lymphomas - correlation with histology and proliferative activity. BrJCancer. 2000;83(9):1161-1167.

73. Grabowska MM, et al. NFI transcription factors interact with FOXA1 to regulate prostatespecific gene expression. Mol Endocrinol. 2014;28(6):949-964.

74. Jia L, et al. Genomic androgen receptor-occupied regions with different functions, defined by histone acetylation, coregulators and transcriptional capacity. PLoS One. 2008;3(11):e3645.

75. Fujiwara T, et al. Discovering hematopoietic mechanisms through genome-wide analysis of GATA factor chromatin occupancy. Mol Cell. 2009;36(4):667-681.

76. Zaret KS, Carroll JS. Pioneer transcription factors: establishing competence for gene expression. Genes Dev. 2011;25(21):2227-2241.

77. Burton LJ, et al. Targeting the nuclear cathepsin L CCAAT displacement protein/cut homeobox transcription factor-epithelial mesenchymal transition pathway in prostate and breast cancer cells with the Z-FY-CHO inhibitor. Mol Cell Biol. 2017;37(5):e00297-16.

78. Wu D, et al. Orphan nuclear receptor TLX functions as a potent suppressor of oncogene-induced senescence in prostate cancer via its transcriptional coregulation of the CDKN1A (p21(WAF1) (/) (CIP1)) and SIRT1 genes. JPathol. 2015;236(1):103-115.

79. Hawksworth D, et al. Overexpression of C-MYC oncogene in prostate cancer predicts biochemical recurrence. Prostate Cancer Prostatic Dis. 2010;13(4):311-315.

80. Koh CM, Bieberich CJ, Dang CV, Nelson WG, Yegnasubramanian S, De Marzo AM. MYC and prostate cancer. Genes Cancer. 2010;1(6):617-628.

81. Murphy DG, Risbridger GP, Bristow RG, Sandhu $\mathrm{S}$. The evolving narrative of DNA repair gene defects: distinguishing indolent from lethal prostate cancer. Eur Urol. 2017;71(5):748-749.

82. Taylor RA, et al. Germline BRCA2 mutations drive prostate cancers with distinct evolutionary trajectories. Nat Commun. 2017;8:13671.

83. Mateo J, et al. DNA repair in prostate cancer: biology and clinical implications. Eur Urol. 2017;71(3):417-425.

84. Pritchard CC, et al. Inherited DNA-repair gene mutations in men with metastatic prostate cancer. N Engl JMed. 2016;375(5):443-453.

85. Pritchard CC, Offit K, Nelson PS. DNA-repair gene mutations in metastatic prostate cancer. N Engl JMed. 2016;375(18):1804-1805.

86. Mateo J, et al. DNA-repair defects and olaparib in metastatic prostate cancer. $N$ Engl J Med. 2015;373(18):1697-1708.

87. Ku SY, et al. Rb1 and Trp53 cooperate to suppress prostate cancer lineage plasticity, metastasis, and antiandrogen resistance. Science. 2017;355(6320):78-83.

88. Xu K, et al. EZH2 oncogenic activity in castrationresistant prostate cancer cells is Polycombindependent. Science. 2012;338(6113):1465-1469.

89. Buenrostro JD, Wu B, Chang HY, Greenleaf WJ. ATAC-seq: a method for assaying chromatin accessibility genome-wide. Curr Protoc Mol Biol. 2015;109:21.29.1-21.29.9. 